\title{
Structural insights and characterization of Human Npas4 protein
}

\author{
Ammad Fahim ${ }^{1}$, Zaira Rehman ${ }^{1}$, Muhammad Faraz Bhatti ${ }^{\text {Corresp.. }}{ }^{1}$, Amjad Ali ${ }^{1}$, Nasar Virk ${ }^{1}$, Amir Rashid $^{2}$, \\ Rehan Zafar Paracha Corresp. 3 \\ ${ }^{1}$ Atta-ur-Rahman School of Applied Biosciences (ASAB), National University of Sciences and Technology (NUST), Islamabad, Pakistan \\ 2 Army Medical College, National University of Medical Sciences, Rawalpindi, Pakistan \\ 3 Research Center for Modeling and Simulation, National University of Sciences \& Technology (NUST), Islamabad, Pakistan
}

Corresponding Authors: Muhammad Faraz Bhatti, Rehan Zafar Paracha

Email address: mfbhatti@asab.nust.edu.pk, rehanzfr@gmail.com

Npas4 is an activity dependent transcription factor which is responsible for gearing the expression of target genes involved in neuro-transmission. Despite the importance of Npas4 in many neuronal diseases, the tertiary structure of Npas4 protein along with its physico-chemical properties is limited. In the current study first we perfomed the phylogenetic analysis of Npas4 and determined the content of hydrophobic, flexible and order-disorder promoting amino acids. The protein binding regions, posttranslational modifications and crystallization propensity of Npas4 was predicted through different insilico methods. The three dimensional model of Npas4 was predicted through LOMET, SPARSKS-X, I-Tasser, RaptorX, MUSTER and Pyhre and the best model was selected on the basis of Ramachandran plot, PROSA, Qmean scores. The best model was then subjected to further refinement though MODREFINER. Finally the interacting partners of Npas4 was identified through STRING database. The phylogenetic analysis showed the human Npas4 gene to be closely related to other primates such as chimpanzees, monkey, gibbon. The physiochemical properties of Npas4 showed that it is an intrinsically disorder protein with $\mathrm{N}$-terminal ordered region. The posttranslational modification analyses indicated absence of acetylation and mannosylation sites. Three potential phosphorylation sites (S108, T130 and T136) were found in PAS A domain whilst a single phosphorylation site (S273) was present in PAS B domain. The predicted tertiary structure of Npas4 showed that bHLH domain and PAS domain possess tertiary structures while the rest of the protein exhibited disorder property. Protein-protein interaction analysis revealed NPas4 interaction with various proteins which are mainly involved in nuclear trafficking of proteins to cytoplasm, activity regulated gene transcription and neurodevelopmental disorders. Moreover the analysis also highlighted direct relation to proteins involved in promoting neuronal survival, plasticity and CAMP responsive element binding protein proteins . The current study helps in understanding the physicochemical properties and reveals the neuro- 
modulatory role of Npas4 in crucial pathways involved in neuronal survival and neural signalling hemostasis. 


\section{Structural insights and characterization of Human Npas4 protein}

2 Ammad Fahim ${ }^{1}$, Zaira Rehman ${ }^{1}$, Muhammad Faraz Bhatti ${ }^{*}$, Amjad Ali ${ }^{1}$, NasarVirk ${ }^{1}$, Amir

3 Rashid $^{3}$, Rehan Zafar Paracha ${ }^{2 *}$

4

51 Atta-ur-Rahman School of Applied Biosciences (ASAB), National University of Sciences and 6 Technology (NUST), Sector H-12, Kashmir Highway, Islamabad 44000, Pakistan

72 Research Center for Modeling and Simulation (RCMS), National University of Sciences and

8 Technology (NUST), Sector H-12, Kashmir Highway, Islamabad 44000, Pakistan

93 Department of Biochemistry, National University of Medical Sciences, Rawalpindi, Pakistan

Authors to whom correspondence should be addressed:

Muhammad Faraz Bhatti ${ }^{*}$ Tel. Office: +92 519085 6136, Fax \#+92 5190856102

1* Atta-ur-Rahman School of Applied Biosciences (ASAB), National University of Sciences and

Technology (NUST), Sector H-12, Kashmir Highway, Islamabad 44000,

15 mfbhatti@asab.nust.edu.pk

Rehan Zafar Paracha ${ }^{2 *}$

$2^{*}$ Research Center for Modeling and Simulation (RCMS), National University of Sciences and

(NUST),

Sector

H-12, Kashmir

Highway,

Islamabad

44000,

Pakistan, rehanzfr@gmail.com

\section{Abstract:}

Npas4 is an activity dependent transcription factor which is responsible for gearing the expression of target genes involved in neuro-transmission. Despite the importance of Npas4 in many neuronal diseases, the tertiary structure of Npas4 protein along with its physico-chemical properties is limited. In the current study first we perfomed the phylogenetic analysis of Npas4 and determined the content of hydrophobic, flexible and order-disorder promoting amino acids. The protein binding regions, posttranslational modifications and crystallization propensity of Npas4 was 
predicted through different in-silico methods. The three dimensional model of Npas4 was predicted through LOMET, SPARSKS-X, I-Tasser, RaptorX, MUSTER and Pyhre and the best model was selected on the basis of Ramachandran plot, PROSA, Qmean scores. The best model was then subjected to further refinement though MODREFINER. Finally the interacting partners of Npas4 was identified through STRING database. The phylogenetic analysis showed the human Npas4 gene to be closely related to other primates such as chimpanzees, monkey, gibbon. The physiochemical properties of Npas4 showed that it is an intrinsically disorder protein with Nterminal ordered region. The posttranslational modification analyses indicated absence of acetylation and mannosylation sites. Three potential phosphorylation sites (S108, T130 and T136) were found in PAS A domain whilst a single phosphorylation site (S273) was present in PAS B domain. The predicted tertiary structure of Npas4 showed that bHLH domain and PAS domain possesstertiary structures while the rest of the protein exhibited disorder property. Protein-protein interaction analysis revealed NPas4 interaction with various proteins which are mainly involved in nuclear trafficking of proteins to cytoplasm, activity regulated gene transcription and neurodevelopmental disorders. Moreover the analysis also highlighted direct relation to proteins involved in promoting neuronal survival, plasticity and cAMP responsive element binding protein proteins. The current study helps in understanding the physicochemical properties and reveals the neuro-modulatory role of Npas4 in crucial pathways involved in neuronal survival and neural signalling hemostasis.

\section{Introduction}

NPas4 belong to the basic helix loop helix/per-arnt-sim (BHLH/PAS) transcription factor family (Shamloo et al. 2006). This family is responsible for gearing the expression of target genes on either positive or negative side (Spiegel et al. 2014). Classically, these transcription factors harbors a DNA binding motif and a dimerisation motif called PAS homology domain (Gu et al. 2000). The word 'PAS' pertains to first three proteins of the domain; period (per), aryl hydrocarbon receptor translocator (arnt) and single minded (SIM)(Ooe et al. 2009). Functionally, Npas4 is categorized as an immediate early gene (IEG), responsible for direct control of large number of activity dependent genes that are capable of altering their expression input on the basis of sensory stimuli they receive (Madabhushi et al. 2015; Sun \& Lin 2016). Npas4 is also known for development of glutaminergic and GABAergic synapses in neurons suggesting its crucial role in neuro-circuitary 
60 homeostasis and memory formation (Spiegel et al. 2014). Deletion of Npas4 may lead to

61 development of several neuronal plasticity disorders and disorganised management of sensory 62 input (Maya-Vetencourt 2013). Previous studies consolidated its functional role in cerebral 63 ischemia, memory developmental disorders and related pathologies like autism spectrum 64 disorders, and neuropsychiatric disorders (Choy et al. 2015a; Choy et al. 2015b; Ebert \&

65 Greenberg 2013; Shepard et al. 2017). Limited studies are available which clearly delineate Npas4 role in regulating its discovered function of neuro-protection and hemostasis in synaptic inter connectivity. The tertiary structure of Npas4 protein along with its physico chemical properties is scarcely available. The current study was mainly aimed to provide the structural insight of Npas4 protein and its possible interaction with other proteins.

\section{Materials and Methods:}

Flow chart of methodology shown in Figure 1.

\subsection{Sequence retrieval and homology search:}

The Npas4 protein sequence was retrieved from UniProt database in FASTA format and was subsequently utilized for homology search using protein BLAST program at NCBI (Blastp). The amino acid sequence of Npas4 was used for homology search using BLASTP at NCBI. One hundred orthologous sequences of Npas4 were retrieved. Out of these 100 sequences 24 full length sequences with homology $>97 \%$ were selected for phylogenetic analysis.

\subsection{Multiple sequence alignment and phylogenetic analysis:}

Multiple sequence alignment of different orthologous sequences were done using ClustalX (version 2.0). All the default paramenters were used for alignment. For Phylogenetic analysis, the tree was constructed by Mega 7 (Kumar et al. 2016) using NJ (Neighbour Joining) method along with1000 bootstraps. The evolutionary distances were computed using the Poisson correction method. All the other parametrs were set as default.

\subsection{Primary sequence analysis}

\subsubsection{Protein order- disorder prediction:}

In order to check whether Npas4 is intrinsically disorder protein or not, predictions were computed using MobiDB web tool and Meta Disorder Web server (Kozlowski \& Bujnicki 2012; Piovesan et al. 2018). MobiDB predicted the disorder region of proteins through the use of six different tools (DisEMBL, ESpritz, GlobPlot, IUPred, Jronn, VSL2b(Piovesan al. 2018). 
91 Metadisorder predicts the disorder regions of proteins through the use of 13 different tools and 92 provides the consensus of all these tools (Kozlowski \& Bujnicki 2012). In order to predict the type 93 of disorder in Npas4, charge hydropathy plot and Cumulative Distribution Function analysis was 94 performed through PONDR server (Xue et al. 2010).

95 3.3.2 Physicochemical analysis of Npas4:

96 The Npas4 primary sequence was used to determine the polarity, mutability, accessibility, 97 bulkiness and refractivity by using Protscale Server on ExPasy platform 98 (http://web.expasy.org/protscale/). The proteins with disorder regions have different composition 99 of flexible and hydrophilic amino acids. The composition of amino acids contribute to flexibility 100 and hydrophilicity was determined through Composition Profiler (http://www.cprofiler.org/).

101 The signal peptide and cleavage sites were predicted on the basis of artificial neural network 102 through SignaIP-4.1 (http:/www.cbs.dtu.dk/services/SignalP/) and SecretomeP 103 (http://www.cbs.dtu.dk/services/SecretomeP/).

104 Prediction of acetylation and mannosylation sites of Npas4 were tallied through NetAcet web server (http://www.cbs.dtu.dk/services/NetAcet) and NetCGlyc web server 106 (http:/www.cbs.dtu.dk/services/NetCGlyc/) respectively, while glycosylation sites were 107 determined through NetOGlyc and NetNGlyc web server (http://www.cbs.dtu.dk/services). 108 Netphos 3.1 server (http:/www.cbs.dtu.dk/services/NetPhos) determined the phosphorylation 109 sites for each Thr, Ser and Tyr residues with 0.5 cut off threshold value. NetPhosK 1.0 server 110 (http://www.cbs.dtu.dk/services/NetPhosK) predicted kinase-specific phosphorylation sites in 111 human NPAS4. For surface accessibility of predicted phosphorylated sites, NetSurfP program 112 (http://www.cbs.dtu.dk/services/NetSurfP) was utilized. Protparam Server 113 (http://web.expasy.org/protparam/) estimated half life, molecular weight, and amino acid 114 composition of Npas4 protein.

\section{3.3.3 Crystallization propensity of NPAS4:}

116 Either fully or partially disorder proteins have little tendency to crystallization. The probability of 117 crystallization of Npas4 was reckoned through PPCpred

118 (http://biomine.cs.vcu.edu/servers/PPCpred/), ParCrys 
120 (http://biomine.cs.vcu.edu/servers/CRYSTALP2/). ParCrys is based on Parzen Window approach

121 that calculate the probalitiy of crystallization based on hydrophobicity, isoelectric point, and 122 frequencies of Ser, Cys, Gly, Phe, Tyr and Met residues (Overton et al. 2008). PPCpred predict 123 the probability of proein crystallization on the basis of four steps of crystallization (production of 124 protein material, purification, crystallization, and diffraction-quality crystallization) and for each 125 step it calclutae the probability and then by combinig all the probabilities it gives the final score 126 of propensity of protein crystallization (Mizianty \& Kurgan 2011).

\subsubsection{Prediction of protein binding regions in disorder npas4:}

It is the property of disorder proteins that they get an ordered conformation upon binding with some globular protein partner. Hence in an attempt to identify these regions ANCHOR web server (http://anchor.enzim.hu/) was used. It identify the regions within disorder segment that cannot fold and form proper stable structure. ANCHOR utilises the IUPred method for prediction (Meszaros et al. 2009).

\subsection{Secondary structure prediction:}

The secondary structure of Npas4 (Accession No: Q8IUM7) was predicted through GOR4 (Garnier et al. 1996), PsiPred (Jones 1999) and JPred3 (Cole et al. 2008).

\subsection{Tertiary structure prediction:}

Npas4 (Uniprot ID: Q8IUM7) was subjected to BLAST search and less than 25\% homology with crytal structure of NPAS3-ARNT complex (5SY7) and NPAS1-ARNT complex (5SY5) was found(figure S1). Due to absence of suitable structural homologue, homology modeling cannot be performed. In the absence of structural homologue, threading protein structure prediction approach was used. Threading is a fold recognition method to predict 3D structure of proteins. I-TASSER ( http://zhanglab.ccmb.med.umich.edu/I-TASSER)

LOMETS (https://zhanglab.ccmb.med.umich.edu/LOMETS/), MUSTER (https://zhanglab.ccmb.med.umich.edu/MUSTER/), $\quad$ SPARSKS-X （http://sparkslab.org/yueyang/server/SPARKS-X/) RaptorX (http://raptorx.uchicago.edu/) and Phyre 2.0 (http://www.sbg.bio.ic.ac.uk/phyre2/html/page.cgi?id=index) was used for three dimensional structure prediction of human Npas4. LOMETS uses local meta threding approach to predict the tertiary structure of protein. It has nine locally installed threding programs and generates an output on the basis of high scoring target-template alignment. I-TASEER is iterative threading program 
150 that build 3D structure by using hierarchical method (Roy et al. 2010). MUSTER is multisource

151 threading program that works by identifying structural templaes from protein data bank and then 152 perform the profile-profile alignment and generates 3D structure (Wu \& Zhang 2008). SPARSKS-

$153 \mathrm{X}$ is fold recognition method to predict 3D structure (Yang et al. 2011). Phyre works by searching 154 of the structural template for the query sequence followed by multiple sequence alignment and 155 then search the hidden markov model of structures to find the best structure (Kelley et al. 2015).

156 3.6 Structure validation of Npas4:

157 Validation of three dimensional structure of NPas4 was performed using PROSA 158 (https://prosa.services.came.sbg.ac.at/prosa.php),

Qmean

159 (http://swissmodel.expasy.org/qmean/cgi/index.cgi) and Ramachandran plot (http://mordred. 160 bioc.cam.ac.uk/_rapper/rampage.php). Ramachandran plot is the visualization of $\psi$ and $\varphi$ 161 torsional angles of protein residues and calculate the residues present in favoured, allowed and 162 outlier region of protein (Lovell et al. 2003). PROSA was used to validate the protein models 163 obtained through different approaches like NMR, X-ray and theoretical calcutations (Wiederstein $164 \&$ Sippl 2007). Qmean provided the estimation of quality of model through Q-score (Benkert et 165 al. 2008).

\section{3.7 Structure refinment:}

167 The validated models were then subjected to refinement using Modrefiner server 168 (https://zhanglab.ccmb.med.umich.edu/ModRefiner/). Modrefiner is an atomic, high resolution 169 protein refinement algorithm (Xu \& Zhang 2011).

\subsection{Protein-protein interaction analysis:}

171 String databse (https://string-db.org/) was used to predict the interaction of Npas4 with other 172 cellular peoteins. STRING is a database of known and predicted protein-protein interactions. In 173 order to get the interacting partners of Npas4 the medium confidence interval value of 0.4 was 174 used and 20 maximum interactions for first shell and 10 maximum interactions for the second shell 175 were used. The interactions may be direct physical interactions or indirect functional associations 176 (Szklarczyk et al. 2017).

177

178

\section{Results:}


179

180

181

182

183

184

185

186

187

188

189

190

191

192

193

194

195

196

197

198

199

200

201

202

203

204

205

206

207

208

209

\subsection{Multiple sequence alignment and phylogenetic analysis:}

Multiple sequence alignment of human Npas4 sequence with others species is shown in Figure S2. The phylogenetic analysis of the aligned sequences of Npas suggests Npas4's evolutionary conservativeness. The analysis of taxonomic classification of all organisms (Supplementary Table S1) revealed human Npas4 to be closely related to other primates including chimpanzees (T.N), monkey, gibbon). The next closest neighbors were rodents (marmart, elephant-shrew, mole, guinea pig). Other relatled organisms to rodents were artiodactyla like camel and alpaca. The farthest neighbors of Npas4 sequences were from rodents include rabbit and pika (Figure 2).

\subsection{Disorder prediction of Npas4:}

The disorder prediction was carried out using Metadisorder and Mobidb. According to Metadisorder and Mobidb results indicated Npas4 is an intrinsically disordered protein. According to Metadisorder plot C-terminal half of the protein showed strong disorder while N-terminal of the protein is ordered (position 15-350 a.a). CDF (cumulative distribution function) analysis showed that NPAS4 is mixture of ordered and disordered regions as it intersects the boundary (Figure 3 ). Primary sequence analysis showed that NPAS4 is enriched in disorder promoting amino acids (Pro, Ser, Glu, Gln, Ala and Gly; Table 1). These amino acids have the tendency to prevent the folding of protein [67-70]. Pro and Ser has the highst probability Amino acid sequence analysis of Npas 4 protein showed that almost $50 \%$ of the protein composed of disorder region. Flexibility analysis of Npas4 was performed using composition profiler and DynaMine server and both servers showed that Npas4 is enriched with flexible amino acids like Pro, Ser and Gln (Figure 4A). Low hydrophobicity and high hydrophilicity promotes disorder in the proteins(Dyson \& Wright 2016). The analysis of according to composition profiler server for suggest NPAS4 to be enriched with hydrophilic amino acids like Gln, Ser, Pro and Thr (Figure 4B).

\subsection{Physico-chemical properties of Npas4:}

The physico-chemical properties were determined by Protscale server (Figure 5). The higher score suggested a higher probability of that particular property of Npas4. The ratio of the side chain volume to the length of an amino acid suggested protein bulkiness and may affect the local structure of a protein. The bulkiness values of Npas4 (Figure 5A) ranges from 9.713 (position 731 aa) to 18.729 (position 295 aa). Hydrophobicity Hopp and wood score (Figure. 5B) and the values were between -0.711 (position 630aa) and 0.422 (position 44 aa). The dipole-dipole intermolecular interactions between the positively and negatively charged particles depicted 
210 polarity as predicted through Zimmerman score (Figure. 5C) The predicted score lies between

2110.272 (position 81 and 82aa) and 34.216 (position 630 aa). This data showed that Npas4 possess

212 polarity. Mutability which is the probability that amino acid will bring a change over a particular

213 evolutionary period of time, was determined through the relative mutability score (Figure. 5D)

214 and the values lies between 46.000 (position 295aa) and 102.889 (position 18 aa) which showed

215 that Npas4 have mutability potential.

216 The signal peptide of Npas4 was computed using SignalP Server. The presence of signal peptide

217 was measured through C- (raw cleavage site score), S- (signal peptide score) and Y-score

218 (combined cleavage site score). S-score is the estimation of possible signal peptide while D-score

219 is the average of mean $\mathrm{S}$ and the max Y-score and it discriminate signal peptide from non-signal

220 peptides. In Npas4, the D-score was 0.450 which was less than the cut off value of 0.5 which

221 showed absence of signal peptide (Figure 6). The secretory nature of Npas4 was predicted through

222 SecretomeP and the NN score which was 0.5.

\subsection{Crystallization propensity of Npas4:}

224 Crystallization propensity was predicted through multiple resources(name) inorder to get a 225 validated prediction. The ParCrys server predicted Npas4 to be 'Recalcitrant to Crystallisation'. 226 According to CRYSTALP2, Npas4 is non-crystallizable with 0.447 confidence. The probability 227 of crystallization was also studied through FDETECT and it gives score of 0.64 which mean this 228 protein is difficult to crystallize. PPCpred provided the crystallization probability score of 0.122 229 for Npas4 while the score above 0.4 means that protein has the ability to crystallize (Mizianty \& 230 Kurgan 2011), suggesting similar difficulty in crystallization

4.5 Protein binding regions in Npas4:

232 Many disorder proteins bind to some other proteins and transformed from disorder to order and 233 thus perform their function(Dyson \& Wright 2016). ANCHOR predicts ten binding sites in the 234 disorder region of Npas4. Out of these ten regions three of them (position 585-599, 662-720, 746235 792) were present in transactivation domain of Npas4 (Table 2).

\section{4.6 Post translation modification sites:}

237 Attempts were made to predict the protein modification sites that could be occurring in Npas4. The 238 results indicated absence of acetylation and mannosylation sites. However, two potential N- 
239 glycosylation sites were found in disorder region of Npas4 at 556 (NPTK) and 671 (NLSL) amino

240 acid positions (Figure 7).

241 A total of 80 O' linked glycosylation sites were present in Npas4. However, only 56 sites were

242 found available for glycosylation according to the GlyCamserver.

243 According to Netphos 3.1 server predictions, 34 threonine phosphorylation sites, 53 serine 244 phosphorylation sites and 4 tyrosine specific phosphorylation sites were present in Npas4 (Figure

245 8). NetsurfP prediction revealed 43 serine, 28 threonine and 3 tyrosine residues were exposed for 246 phosphorylation. Supplementary table S2 showed the 24 Serine, 13 Threonine and 1 Tyrosine 247 kinase specific sites in Npas4. Two phosphorylation sites present (S38, S44) in bHLH domain and 248 three sites (S98, S100 and T136) were observed in PASA domain while 1 site (S273) seen in PAS 249 B domain. Six Ser and four Thr phosphorylation sites present in disordered transactivation domain 250 (Table 3).

251 Different physiological parameters of Npas4 were predicted trough Protparam server (Table 4).

252 4.7 Secondary structure of Npas4:

253 The secondary structure of Npas4 showed that the protein consists of alpha helices, coils and beta 254 sheet (Figure S3).

\subsection{Tertiary structure of Npas4:}

256 The three dimensional structure of NPAS4 protein has not been determined to date. Due to the 257 absence of suitable structural template holmology modeling can not be used (Figure S1). In order 258 to get the high quality structure of Npas4 ab-initio and threading approaches were used. By using 259 these approaches five models were generated through I-Tasser, ten models, one model from 260 RaptorX, ten models from MUSTER, one model from Phyre and ten models from SPARSK-X 261 (Table 5). All the 37 models were then subjected to validation through PROSA, Qmean and 262 Ramachandran plot. The models with $96 \%$ residues in favoured region were further selected for 263 refinement (Table 6 Figure 9). All models suggested that Npas4 is a disordered protein with 264 ordered bHLH and PAS domain. Based on Qmean, PROSA Z-score and ramachandran scores, 265 model 9 generated LOMET was the best predicted three dimensional structure of Npas4. The 266 possible phosphorylation sites in PASA and PASB domain of Npas4 is shown in Figure 10.

\section{4.9 Protein-protein interaction analysis of Npas4:}

268 To study interaction of Npas4 with other proteins, STRING database v10 (Szklarczyk et al. 2015) 269 was used (Figure 11). The results suggested Npas4 interaction with ARNT (Aryl hydrocarbon 
270 receptor nuclear translocator); NXF2B (nuclear RNA export factor 2B); NXF2 (nuclear export 271 factor 2); NXF3 (nuclear export factor 3); NDNL2 (necdin-like 2); MAGEF1 (melanoma antigen

272 family F, 1); C3orf58 (chromosome 3 open reading frame 58); NXF1 (nuclear RNA export factor 273 1); NUP107 (nucleoporin 107KDa); CSAG1 (Chondrosarcoma associated gene 1 ); MAGEF1 274 (Melanoma antigen family F, 1 ); SLC9A6 (Solute carrier family 9, subfamily A (NHE6, cation 275 proton antiporter 6), member 6); MAGED2 (Melanoma antigen family D, 2 ); SLC9A9 (Solute 276 carrier family 9, subfamily A (NHE9, cation proton antiporter 9), member 9); MAGEL2 (MAGE277 like 2); SSX2B (Synovial sarcoma, X breakpoint 2B) SLC9A1 (Solute carrier family 9, subfamily 278 A (NHE1, cation proton antiporter 1), member 1); CREB1 (cAMP responsive element binding 279 protein 1); MAGEA1 (Melanoma antigen family A, 1 ) \& EPAS1 (Endothelial PAS domain280 containing protein 1 ( also known as hypoxia-inducible factor-2alpha (HIF-2alpha).

\section{Discussion:}

282

283

284

285

286

287

288

289

290

291

292

293

294

295

296

297

298

299

The Npas4 gene has been in debate for more than a decade, however its structural information is scarcely available. Npas4 expression is primarily traced to neural cells, where it has been reported to be an important contributor of dendritic growth in phases of neuronal development and for modulating limbic patterning and function (Moser et al. 2004). Recently, it has been reported to be also expressed in pancreatic cells (Speckmann et al. 2016). Functionally, Npas4 is an immediate early gene, which under activation, triggers the activation of battery of genes involved in regulating brain plasticity and cognition, attributed mainly to its interaction with number of transcription factors. Npas4 belongs to bHLH-PAS family of transcription factors and the other Npas members (1 and 3) are also linked to numerous psychiatric disorders namely autism, bipolar disorders, schizophrenia and depressive disorders (Adachi 2014; Kamnasaran et al. 2003). A simulated dimerised structure of Npas4 with ARNT Iwas reported before to discuss the potential implication of gene variants (Bersten et al. 2014). Current findings addresses physicochemical properties on human Npas4 protein, with a plausible 3D model, providing useful information about amino acid characterstics and possible identification of interactable proteins.

Human Npas4 is located on human chromosome 11 reference genomic contig NC000011.10, mapping to the chromosomal position 11q13.2. It possess 11 exons that encodes 2406 bp mRNA which translates into 802 amino acid long protein with $87.1 \mathrm{KDa}$ molecular weight (https://www.ncbi.nlm.nih.gov/gene/266743). 
300 In the current study the functional characterization of $\mathrm{Npas} 4$ has been done using in silico

301 approaches. The MSA and phylogentic analysis showed that Npas4 is evolutionry conserved, 302 with reflecting highest homology to primates as Chimpanzee and Monkey.

303 Npas4 does not have any signal peptide hence it may not being classically secreted extracellularly.

304 The NN score of SecretomeP also verifes Npas4 to be a non-classicaly secreted protein.

305 The physicochemical and fuctional properties of proteins are affected by posttranslational 306 modifications. Phosphorylation, acetylation and glycosylation are the most common type of 307 posttranslational modifications of proteins (Koh et al. 2012).

308 Acetylation is one of the major post-translational modifications (PTMs) and is important in 309 determining the cellular localization of proteins (Qin et al. 2011). Theres is no acetylation site 310 present in Npas4 protein.

311 Glycosylation, the addition of glycosyl moiety to protein, is the most common post-translational 312 modification in eukaryotes. N-linked and O-linked glycosylations are common while C-linked 313 (mannosylation) is rare (Koh et al. 2012). No C-linked glycosylation sites were present in Npas4.

314 In Npas4, 2 N-linked glycosylation sites were present but these sites were not available for 315 glycosylation due to the absence of signal peptide.

316 Phosphorylation is another important feature contributing in PTM. Phosphorylation changes the 317 conformation of protein making it active, deactive or modifying its function (Raghava et al. 2014). 318 In Npas4 there were 90 potential phosphorylation sites which include 34 Threonine, 53 Serine and 3194 Tyrosine phosphorylation sites. But not all the sites were available for phosphorylation. There 320 were 24 Serine, 13 Theronine and 1 Tyrosine site available for phosphorylation. The important 321 phosphorylation sites present in PAS A and PAS B domain were found at position 130, 136 and 322273 respectively. Further experimental studies are needed to explore the role of these sites in Npas 4 323 function.

324

325

326

327

328

329 330

Prediction analysis in the current study suggested presence of intrinsically disordered protein residues (IDRs) in Npas4 sequence at the $\mathrm{C}$ terminal, that can have profound effect on its functional versatility. Conventionally, proteins at physiological temperature exhibit a particular conformational ensemble based on optimal thermal accessibility of the ensemble to various molecular crowders and interacting proteins (Wright \& Dyson 1999). However, intrinsically disordered proteins or intrinsically disordered protein residues (IDRs) in a particular protein 
331 enables the protein to interconvert among various topological conformations (Dyson \& Wright 332 2005). These IDRs therefore, can provide Npas4, ease of flexibility to engage multiple targets. 333 Moreover, IDRs can also aid in interactions by efficient utilization of less residues. This will 334 enhance spontaneous disassociation or displacement in neuronal pathways. Previously it has been 335 reported that structural homogeneity exhibited by IDRs is deficient which results from varied 336 composition of Gly and Pro charged residues (Wright \& Dyson 2015), as is the case of Npas4, 337 which may subsequently resulted in decreased protein folding. This can further be implicated to 338 enhance conformational plasticity of Npas4 which may help in behaving differently to various 339 transcription factors and enhancing its functional repertoire. The presence of IDRs C-terminal 340 regulatory domain have also been reported in other Bhlh proteins (Fribourgh \& Partch 2017)

341 Moreover, previous reported behavior of IDRs suggest that the presence of more charged residues 342 enhances electrostatic interactions which augments the propensity of post translational 343 modifications (Hofmann et al. 2012; Mao et al. 2010). This subsequently resulted in altered 344 binding affinities and increased range of structural heterogeneity derived from disorder to order transitions altering protein compactness. The simultaneous consideration of Npas4 as an 'immediate early gene', alongwith all the predicted and implied consequences of harboring IDRs in its sequence. The PAS domains carrying multi-ligand binding properties can aid Npas4 to impart its role as a 'hub protein' in forming various complexes and key mediator in neuronal signaling.

The presence of IDR within Npas4 sequence can pose difficulty in sculpting protein structure through X-ray crystallography which may be attributable to hindrances caused by the crystal packing forces from promiscuous conformations of disordered regions (Wells et al. 2008). This could be one of the reasons that detrermination of Npas4 crystallization propensity was reported to be on very low scores from all servers used.

The function of protein can be best predicted if we have insight about the protein tertiary structure. The Npas4 sequence have less than 25\% homology with crystal structure of PAS domain of Npas3 an Npas1. In the absence of any structural template, ab-initio modeling approach for Npas4 structure prediction was opted. The modeled structure was composed of alpha helices and B sheets 
360 in the bHLH domain and PAS A and B domains, while the transactivation domain does not passess

361 any tertiary structure due to the presence of IDRs. Due to the presence of proper tertiary structure 362 of PAS domain it involved in heterodimarization with its partner (ARNT). Current study of amino 363 acid properties suggested preponderance of hydrophobic amino acids in both PAS A and B 364 domains. These findings are in accordance with other bHLH proteins like Npas1, Npas3, BMAL1 365 and CLOCK where PAS domains are harboring internal hydrophobic cavities (Wu et al. 2016).

366

367

368

369

370

371

372

373

374

375

376

377

378

379

380

381

382

383

384

385

386

387

388

389

390

The Npas4 was also subjected to prediction of interacting proteins and it reflected its interaction with ARNT, which is its main partner for dimerization. The STRING analysis also showed some important interactions. The analysis showed that Npas4 functional interaction can be divided into 4 clusters or groups. Cluster 1 involves proteins which are responsible for forming large protein complex (the SMC5-SMC6 complex). This protein complex is responsible for inducing double stranded DNA breaks (DSB) under neuronal stimulation, which is crucial for the expression downstream promoters such as BDNF(Madabhushi et al. 2015). Through its interaction with CDK5, which has a key role in BDNF induced dendrite development, it implicates the crucial role of Npas4 in memory plasticity through regulating the balance of inhibition in neuronal synapses. This interaction may also be related to Rett Syndrome, a disorder in which activity dependent $B D N F$ transcription is hampered(Liang et al. 2015). Cluster 2 (Figure 8) suggested Npas4 interaction to Nuclear RNA export factor (NXF) proteins, which are RNA binding proteins, with various paralogs reported to be found in dendritic granules (Mamon, Ginanova et. al.2017), suggesting Npas4's active involvement in the nuclear export of different mRNA and translational control over different proteins. Cluster 3 proteins reported to have altered expression in autism spectrum disorders (ASD), that may consequently result from knockdown of activity regulated gene transcription mediated by Npas4 (Morrow et al. 2008). Cluster 4 represented interaction of Npas4 interaction with cyclic AMP responsive element binding (CREB) proteins. These proteins under phosphorylated state can alter chromatin structure by histones acetylation which speeds up the RNA polymerase II recruitement, trigerring the gene transcriptional programs involved in synapse development (Cohen \& Greenberg 2008; Greer \& Greenberg 2008). The CREB alongwith Npas4 is also involved in regulating inhibitory synapses of excitatoty neurons by activating BDNF transcription (Hong et al. 2008; Lin et al. 2008). Interstingly, we found three binding sites in the transactivation domain which falls in the region of IDR. This information provides a plausible rationale that CREB may be interacting with any of these residues from the transactvation domain 
391 through interaction with transcriptional coactivator, CBP which is known to cause such interaction

392 through IDRs (Dyson \& Wright 2016).

393

394

395

396

397

398

399

400

401

402

403

404

405

406

407

408

409

410

411

412

413

414

415

416

417

418

419

\section{Conclusion:}

Npas4 is an intrinsically disorder protein with ordered bHLH and PAS domain. The amino acid sequence analysis showed that Npas4 have high proportion of flexible and hydrophobic amino acids that promote the disorder properties of proteins. The model 9 predicted through LOMET is the best structure of Npas4 and can be used for further analysis. As this protein is difficult to crystalize so inorder to determine its tertiary structure we can use NMRand other related techniques. It has strong interaction with NXF 2B and NXF 3 proteins which implicates its potential role in cytoplasmic export of proteins from nucleus, thus influencing protein translation. Current study also elucidates Npas4 activation with CREB proteins, suggesting Npas4 reliance on $\mathrm{Ca}^{2+}$ dependent kinases (CDKs). The information compiled in this research can serve as useful information for identifying new drug targets, which can modulate synaptic hemostasis in neuropshychiatric and neuro-developmental disorders. Moreover, possible interaction with Nuclear RNA export factor (NXF) protein family role identification needs further elaboration. The results reported by our study are more of structural and theoretical in nature, however, they may help in biophysical studies, NMR and crystallographic studies directed towards Npas4. Moreover, the considerations of results may help in future studies designed to understand binding interactions of Npas4.

\section{Abbreviations:}

ARNT (Aryl hydrocarbon receptor nuclear translocator);

NXF2B (nuclear RNA export factor 2B);

NXF2 (nuclear export factor 2);

NXF3 (nuclear export factor 3);

NDNL2 (necdin-like 2);

MAGEF1 (melanoma antigen family F, 1);

C3orf58 (chromosome 3 open reading frame 58);

NXF1 (nuclear RNA export factor 1);

NUP107 (nucleoporin 107KDa); 
420 CSAG1 (Chondrosarcoma associated gene 1 );

421 MAGEF1 (Melanoma antigen family F, 1 );

422 SLC9A6 (Solute carrier family 9, subfamily A

423 NHE6, cation proton antiporter 6), member 6);

424 MAGED2 (Melanoma antigen family D, 2 );

425 SLC9A9 (Solute carrier family 9, subfamily A

426 NHE9, cation proton antiporter 9), member 9);

427 MAGEL2 (MAGE-like 2);

428 SSX2B (Synovial sarcoma, $X$ breakpoint 2B)

429 SLC9A1 (Solute carrier family 9, subfamily A

430 NHE1, cation proton antiporter 1), member 1);

431 CREB1 (cAMP responsive element binding protein 1);

432 MAGEA1 (Melanoma antigen family A, 1 ) \&

433 EPAS1 (Endothelial PAS domain-containing protein 1 ( also known as hypoxia-inducible factor-

434 2alpha (HIF-2alpha).

435

436

437

438

439

440

441

442

443

444

445

446

447

448

449

450

451

452

453

454

455

\section{References:}

Adachi N. 2014. New insight in expression, transport, and secretion of brain-derived neurotrophic factor: Implications in brain-related diseases. World Journal of Biological Chemistry 5:409. 10.4331/wjbc.v5.i4.409

Benkert P, Tosatto SC, and Schomburg D. 2008. QMEAN: A comprehensive scoring function for model quality assessment. Proteins 71:261-277. 10.1002/prot.21715

Bersten DC, Bruning JB, Peet DJ, and Whitelaw ML. 2014. Human variants in the neuronal basic helix-loophelix/Per-Arnt-Sim (bHLH/PAS) transcription factor complex NPAS4/ARNT2 disrupt function. PLOS ONE 9:e85768. 10.1371/journal.pone.0085768

PONE-D-13-37530 [pii]

Choy F, Klarić T, Koblar S, and Lewis M. 2015a. The Role of the Neuroprotective Factor Npas4 in Cerebral Ischemia. International Journal of Molecular Sciences 16:29011-29028. 10.3390/ijms161226144

Choy FC, Klarić TS, Leong WK, Koblar SA, and Lewis MD. 2015b. Reduction of the neuroprotective transcription factor Npas4 results in increased neuronal necrosis, inflammation and brain lesion size following ischaemia. Journal of Cerebral Blood Flow \& Metabolism 36:1449-1463. 10.1177/0271678x15606146

Cohen S, and Greenberg ME. 2008. Communication Between the Synapse and the Nucleus in Neuronal Development, Plasticity, and Disease. Annual Review of Cell and Developmental Biology 24:183209. 10.1146/annurev.cellbio.24.110707.175235

Cole C, Barber JD, and Barton GJ. 2008. The Jpred 3 secondary structure prediction server. Nucleic Acids Research 36:W197-W201. 10.1093/nar/gkn238 
456

457

458

459

460

461

462

463

464

465

466

467

468

469

470

471

472

473

474

475

476

477

478

479

480

481

482

483

484

485

486

487

488

489

490

491

492

493

494

495

496

497
Dyson HJ, and Wright PE. 2005. Intrinsically unstructured proteins and their functions. Nat Rev Mol Cell Biol 6:197-208. nrm1589 [pii]

\section{$10.1038 / \mathrm{nrm} 1589$}

Dyson HJ, and Wright PE. 2016. Role of Intrinsic Protein Disorder in the Function and Interactions of the Transcriptional Coactivators CREB-binding Protein (CBP) and p300. J Biol Chem 291:6714-6722. 10.1074/jbc.R115.692020

R115.692020 [pii]

Ebert DH, and Greenberg ME. 2013. Activity-dependent neuronal signalling and autism spectrum disorder. Nature 493:327-337. 10.1038/nature11860

Fribourgh JL, and Partch CL. 2017. Assembly and function of bHLH-PAS complexes. Proc Natl Acad Sci U S A 114:5330-5332. 10.1073/pnas.1705408114

1705408114 [pii]

Garnier J, Gibrat JF, and Robson B. 1996. GOR method for predicting protein secondary structure from amino acid sequence. Methods Enzymol 266:540-553. S0076-6879(96)66034-0 [pii]

Greer PL, and Greenberg ME. 2008. From Synapse to Nucleus: Calcium-Dependent Gene Transcription in the Control of Synapse Development and Function. Neuron 59:846-860. 10.1016/j.neuron.2008.09.002

Gu YZ, Hogenesch JB, and Bradfield CA. 2000. The PAS superfamily: sensors of environmental and developmental signals. Annu Rev Pharmacol Toxicol 40:519-561. 10.1146/annurev.pharmtox.40.1.519

Hofmann H, Soranno A, Borgia A, Gast K, Nettels D, and Schuler B. 2012. Polymer scaling laws of unfolded and intrinsically disordered proteins quantified with single-molecule spectroscopy. Proc Natl Acad Sci U S A 109:16155-16160. 10.1073/pnas.1207719109

1207719109 [pii]

Hong EJ, McCord AE, and Greenberg ME. 2008. A Biological Function for the Neuronal Activity-Dependent Component of Bdnf Transcription in the Development of Cortical Inhibition. Neuron 60:610-624. 10.1016/j.neuron.2008.09.024

Jones DT. 1999. Protein secondary structure prediction based on position-specific scoring matrices. J Mol Biol 292:195-202. 10.1006/jmbi.1999.3091

S0022-2836(99)93091-7 [pii]

Kamnasaran D, Muir WJ, Ferguson-Smith MA, and Cox DW. 2003. Disruption of the neuronal PAS3 gene in a family affected with schizophrenia. J Med Genet 40:325-332.

Kelley LA, Mezulis S, Yates CM, Wass MN, and Sternberg MJ. 2015. The Phyre2 web portal for protein modeling, prediction and analysis. Nat Protoc 10:845-858. 10.1038/nprot.2015.053

nprot.2015.053 [pii]

Koh GCKW, Porras P, Aranda B, Hermjakob H, and Orchard SE. 2012. Analyzing Protein-Protein Interaction Networks. Journal of Proteome Research 11:2014-2031. 10.1021/pr201211w

Kozlowski LP, and Bujnicki JM. 2012. MetaDisorder: a meta-server for the prediction of intrinsic disorder in proteins. BMC Bioinformatics 13:111. 10.1186/1471-2105-13-111

1471-2105-13-111 [pii]

Kumar S, Stecher G, and Tamura K. 2016. MEGA7: Molecular Evolutionary Genetics Analysis Version 7.0 for Bigger Datasets. Molecular Biology and Evolution 33:1870-1874. 10.1093/molbev/msw054 
498

499

500

501

502

503

504

505

506

507

508

509

510

511

512

513

514

515

516

517

518

519

520

521

522

523

524

525

526

527

528

529

530

531

532

533

534

535

536

537

538

539

540

541

Liang Z, Ye T, Zhou X, Lai KO, Fu AKY, and Ip NY. 2015. Cdk5 Regulates Activity-Dependent Gene Expression and Dendrite Development. Journal of Neuroscience 35:15127-15134. 10.1523/jneurosci.144315.2015

Lin Y, Bloodgood BL, Hauser JL, Lapan AD, Koon AC, Kim T-K, Hu LS, Malik AN, and Greenberg ME. 2008. Activity-dependent regulation of inhibitory synapse development by Npas4. Nature 455:11981204. 10.1038/nature07319

Lovell SC, Davis IW, Arendall WB, 3rd, de Bakker PI, Word JM, Prisant MG, Richardson JS, and Richardson DC. 2003. Structure validation by Calpha geometry: phi,psi and Cbeta deviation. Proteins 50:437450. 10.1002/prot.10286

Madabhushi R, Gao F, Pfenning Andreas R, Pan L, Yamakawa S, Seo J, Rueda R, Phan TX, Yamakawa H, Pao P-C, Stott Ryan T, Gjoneska E, Nott A, Cho S, Kellis M, and Tsai L-H. 2015. Activity-Induced DNA Breaks Govern the Expression of Neuronal Early-Response Genes. Cell 161:1592-1605. 10.1016/j.cell.2015.05.032

Mao AH, Crick SL, Vitalis A, Chicoine CL, and Pappu RV. 2010. Net charge per residue modulates conformational ensembles of intrinsically disordered proteins. Proc Natl Acad Sci U S A 107:81838188. 10.1073/pnas.0911107107

\section{7 [pii]}

Maya-Vetencourt JF. 2013. Activity-DependentNPAS4Expression and the Regulation of Gene Programs Underlying Plasticity in the Central Nervous System. Neural Plasticity 2013:1-12. $10.1155 / 2013 / 683909$

Meszaros B, Simon I, and Dosztanyi Z. 2009. Prediction of protein binding regions in disordered proteins. PLoS Comput Biol 5:e1000376. 10.1371/journal.pcbi.1000376

Mizianty MJ, and Kurgan L. 2011. Sequence-based prediction of protein crystallization, purification and production propensity. Bioinformatics 27:i24-33. 10.1093/bioinformatics/btr229

btr229 [pii]

Morrow EM, Yoo SY, Flavell SW, Kim TK, Lin Y, Hill RS, Mukaddes NM, Balkhy S, Gascon G, Hashmi A, AlSaad S, Ware J, Joseph RM, Greenblatt R, Gleason D, Ertelt JA, Apse KA, Bodell A, Partlow JN, Barry B, Yao H, Markianos K, Ferland RJ, Greenberg ME, and Walsh CA. 2008. Identifying Autism Loci and Genes by Tracing Recent Shared Ancestry. Science 321:218-223. 10.1126/science.1157657

Moser M, Knoth R, Bode C, and Patterson C. 2004. LE-PAS, a novel Arnt-dependent HLH-PAS protein, is expressed in limbic tissues and transactivates the CNS midline enhancer element. Molecular Brain Research 128:141-149. 10.1016/j.molbrainres.2004.06.023

Ooe N, Saito K, and Kaneko H. 2009. Characterization of functional heterodimer partners in brain for a bHLH-PAS factor NXF. Biochimica et Biophysica Acta (BBA) - Gene Regulatory Mechanisms 1789:192-197. 10.1016/j.bbagrm.2009.01.003

Overton IM, Padovani G, Girolami MA, and Barton GJ. 2008. ParCrys: a Parzen window density estimation approach to protein crystallization propensity prediction. Bioinformatics 24:901-907. 10.1093/bioinformatics/btn055

btn055 [pii]

Piovesan D, Tabaro F, Paladin L, Necci M, Micetic I, Camilloni C, Davey N, Dosztanyi Z, Meszaros B, Monzon AM, Parisi G, Schad E, Sormanni P, Tompa P, Vendruscolo M, Vranken WF, and Tosatto SCE. 2018. MobiDB 3.0: more annotations for intrinsic disorder, conformational diversity and interactions in proteins. Nucleic Acids Res 46:D471-D476. 10.1093/nar/gkx1071

4612964 [pii] 
542

543

544

545

546

547

548

549

550

551

552

553

554

555

556

557

558

559

560

561

562

563

564

565

566

567

568

569

570

571

572

573

574

575

576

577

578

579

580

581

582

583

584

585
Qin S, Pang X, and Zhou H-X. 2011. Automated Prediction of Protein Association Rate Constants. Structure 19:1744-1751. 10.1016/j.str.2011.10.015

Raghava GPS, Yu C-S, Cheng C-W, Su W-C, Chang K-C, Huang S-W, Hwang J-K, and Lu C-H. 2014. CELLO2GO: A Web Server for Protein subCELlular LOcalization Prediction with Functional Gene Ontology Annotation. PLoS ONE 9:e99368. 10.1371/journal.pone.0099368

Roy A, Kucukural A, and Zhang Y. 2010. I-TASSER: a unified platform for automated protein structure and function prediction. Nat Protoc 5:725-738. 10.1038/nprot.2010.5

nprot.2010.5 [pii]

Shamloo M, Soriano L, von Schack D, Rickhag M, Chin DJ, Gonzalez-Zulueta M, Gido G, Urfer R, Wieloch T, and Nikolich K. 2006. Npas4, a novel helix-loop-helix PAS domain protein, is regulated in response to cerebral ischemia. European Journal of Neuroscience 24:2705-2720. 10.1111/j.14609568.2006.05172.x

Shepard R, Heslin K, and Coutellier L. 2017. The transcription factor Npas4 contributes to adolescent development of prefrontal inhibitory circuits, and to cognitive and emotional functions: Implications for neuropsychiatric disorders. Neurobiology of Disease 99:36-46. 10.1016/j.nbd.2016.12.012

Speckmann T, Sabatini PV, Nian C, Smith RG, and Lynn FC. 2016. Npas4 Transcription Factor Expression Is Regulated by Calcium Signaling Pathways and Prevents Tacrolimus-induced Cytotoxicity in Pancreatic Beta Cells. J Biol Chem 291:2682-2695. 10.1074/jbc.M115.704098

M115.704098 [pii]

Spiegel I, Mardinly Alan R, Gabel Harrison W, Bazinet Jeremy E, Couch Cameron H, Tzeng Christopher P, Harmin David A, and Greenberg Michael E. 2014. Npas4 Regulates Excitatory-Inhibitory Balance within Neural Circuits through Cell-Type-Specific Gene Programs. Cell 157:1216-1229. 10.1016/j.cell.2014.03.058

Sun X, and Lin Y. 2016. Npas4: Linking Neuronal Activity to Memory. Trends in Neurosciences 39:264-275. 10.1016/j.tins.2016.02.003

Szklarczyk D, Franceschini A, Wyder S, Forslund K, Heller D, Huerta-Cepas J, Simonovic M, Roth A, Santos A, Tsafou KP, Kuhn M, Bork P, Jensen LJ, and von Mering C. 2015. STRING v10: protein-protein interaction networks, integrated over the tree of life. Nucleic Acids Research 43:D447-D452. 10.1093/nar/gku1003

Szklarczyk D, Morris JH, Cook H, Kuhn M, Wyder S, Simonovic M, Santos A, Doncheva NT, Roth A, Bork P, Jensen LJ, and von Mering C. 2017. The STRING database in 2017: quality-controlled proteinprotein association networks, made broadly accessible. Nucleic Acids Res 45:D362-D368. 10.1093/nar/gkw937

gkw937 [pii]

Wells M, Tidow H, Rutherford TJ, Markwick P, Jensen MR, Mylonas E, Svergun DI, Blackledge M, and Fersht AR. 2008. Structure of tumor suppressor p53 and its intrinsically disordered N-terminal transactivation domain. Proc Natl Acad Sci U S A 105:5762-5767. 10.1073/pnas.0801353105

0801353105 [pii]

Wiederstein M, and Sippl MJ. 2007. ProSA-web: interactive web service for the recognition of errors in three-dimensional structures of proteins. Nucleic Acids Res 35:W407-410. gkm290 [pii]

10.1093/nar/gkm290

Wright PE, and Dyson HJ. 1999. Intrinsically unstructured proteins: re-assessing the protein structurefunction paradigm. J Mol Biol 293:321-331. 10.1006/jmbi.1999.3110 
586

587

588

589

590

591

592

593

594

595

596

597

598

599

600

601

602

603

604

605

606

607

608

609

S0022-2836(99)93110-8 [pii]

Wright PE, and Dyson HJ. 2015. Intrinsically disordered proteins in cellular signalling and regulation. Nat Rev Mol Cell Biol 16:18-29. 10.1038/nrm3920

nrm3920 [pii]

Wu D, Su X, Potluri N, Kim Y, and Rastinejad F. 2016. NPAS1-ARNT and NPAS3-ARNT crystal structures implicate the bHLH-PAS family as multi-ligand binding transcription factors. Elife 5. 10.7554/eLife. 18790

e18790 [pii]

Wu S, and Zhang Y. 2008. MUSTER: Improving protein sequence profile-profile alignments by using multiple sources of structure information. Proteins 72:547-556. 10.1002/prot.21945

Xu D, and Zhang Y. 2011. Improving the physical realism and structural accuracy of protein models by a two-step atomic-level energy minimization. Biophys J 101:2525-2534. 10.1016/j.bpj.2011.10.024

S0006-3495(11)01245-8 [pii]

Xue B, Dunbrack RL, Williams RW, Dunker AK, and Uversky VN. 2010. PONDR-FIT: a meta-predictor of intrinsically disordered amino acids. Biochim Biophys Acta 1804:996-1010. 10.1016/j.bbapap.2010.01.011

S1570-9639(10)00013-0 [pii]

Yang Y, Faraggi E, Zhao H, and Zhou Y. 2011. Improving protein fold recognition and template-based modeling by employing probabilistic-based matching between predicted one-dimensional structural properties of query and corresponding native properties of templates. Bioinformatics 27:2076-2082. 10.1093/bioinformatics/btr350

btr350 [pii]

.


Figure 1

Flowchart used for functional annotation of Npas4.

Sequence retrieval of Npas4 followed by homology search and multiple sequence alignment and phylogenetic analysis. Assessment of physiological properties, posttranslational modifications of Npas4 were predicted using primary sequence. Subsequently, secondary and tertiary structure were predicted through Ab-initio modeling.

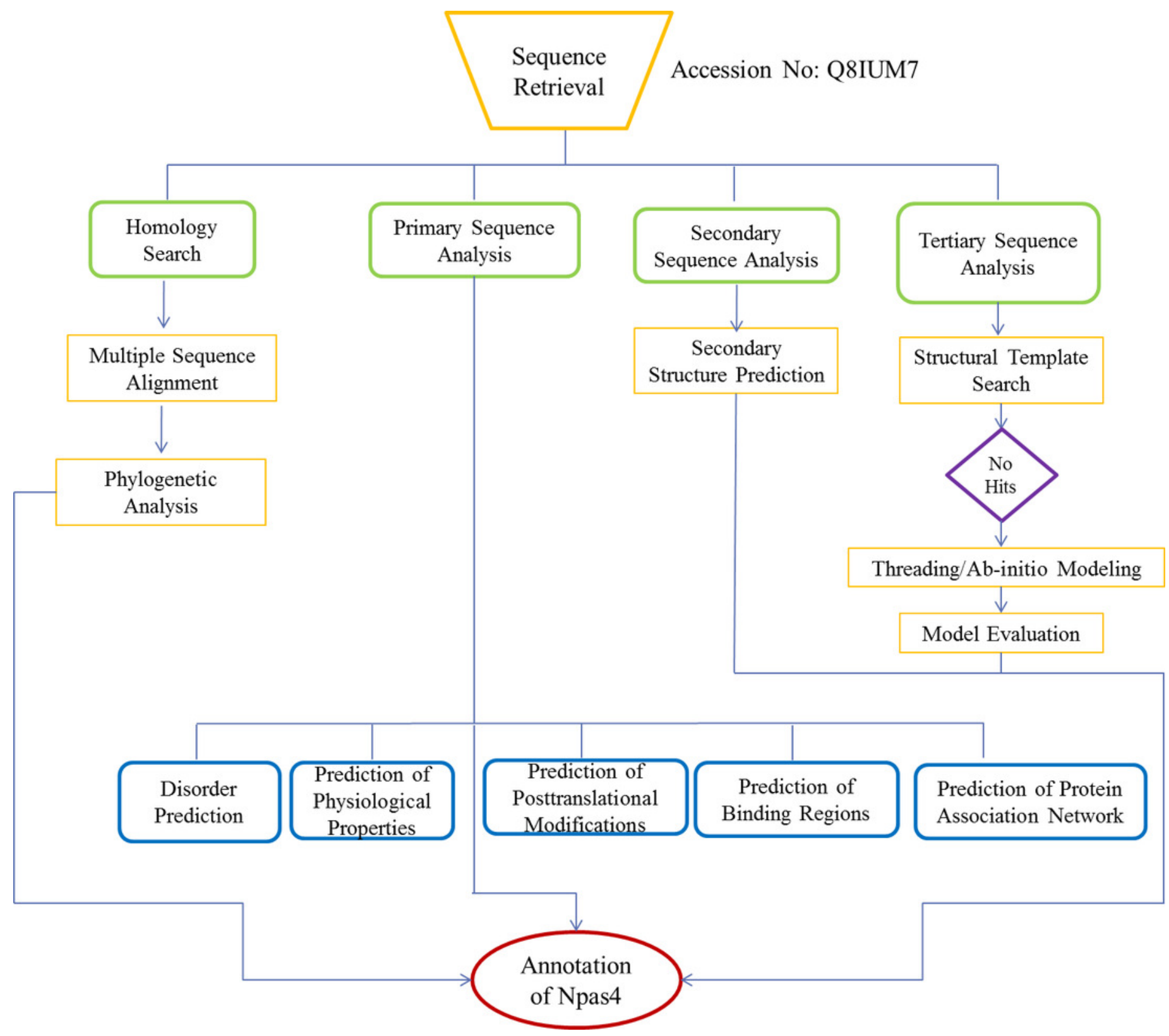




\section{Figure 2}

The phylogenetic tree of human Npas4 generated by NJ method with 1000 bootstrap value.

The tree showed that human Npas4 closest homology with other primates like Chimpanzee (Pan troglodytes), Monkey (Rhinopithecus bieti) and Gibbons (Nomascus leucogenys) while the farthest orthologs were Rabbit (Oryctolagus cuniculus) and Pika (Ochotona princeps)

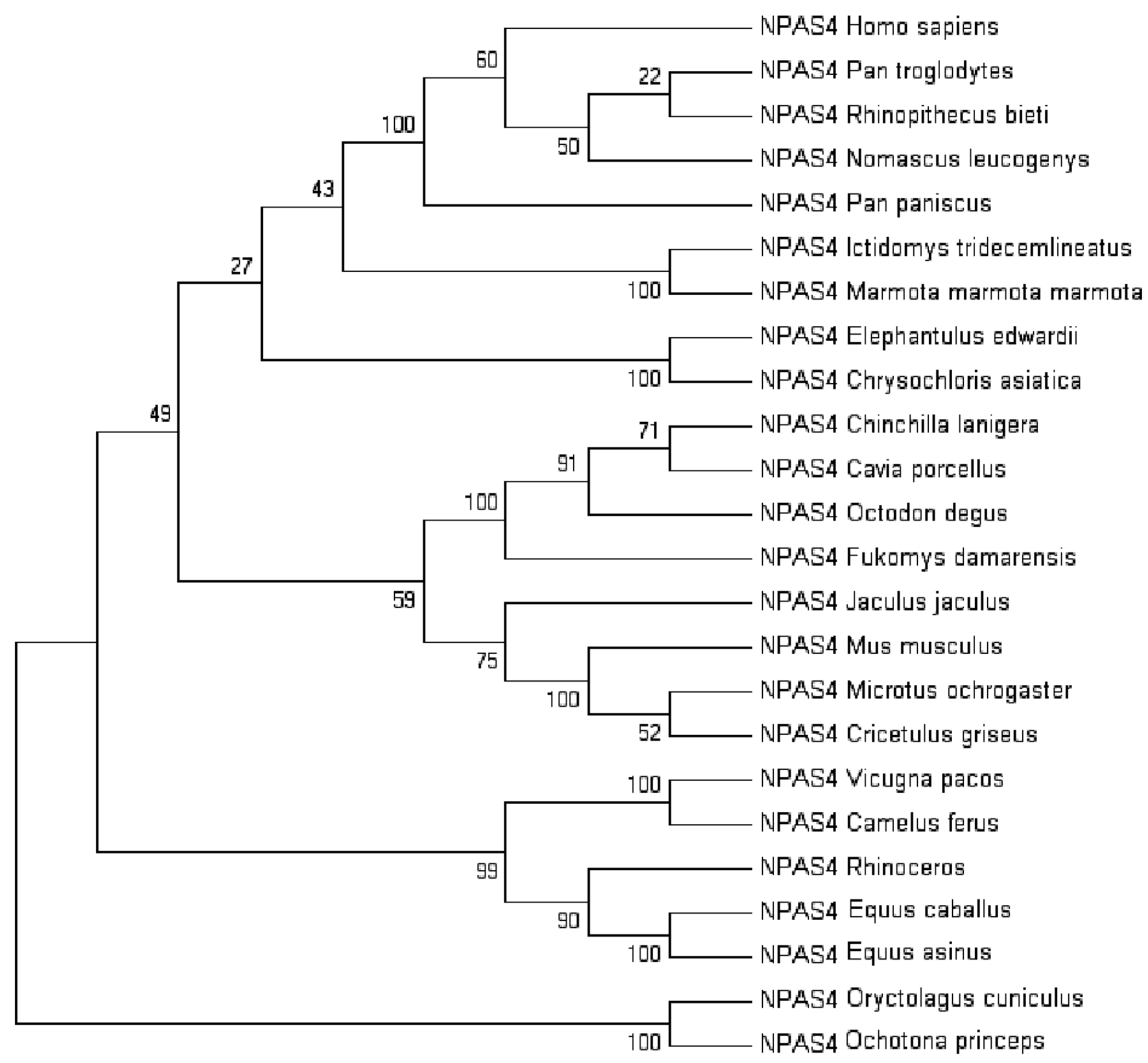




\section{Figure 3}

Disorder prediction of Npas4.

(A) Metadisorder server predicts the disorder regions in Npas4 primary sequence. The plot shows the prediction of three versions of Metadisorder. The value above 0.5 shows disorder region from position 350 to 802. (B) CDF plot of Npas4 calculated through PONDR server. It predicts either order or disorder proteins. Black line is the boundary, the proteins above the boundary are predicted to be ordered, below the boundary are disordered while the proteins that intersect the boundary is the mixture of order and disorder. According to CDF, Npas4 is mixture of order and disorder.

$\mathbf{A}$

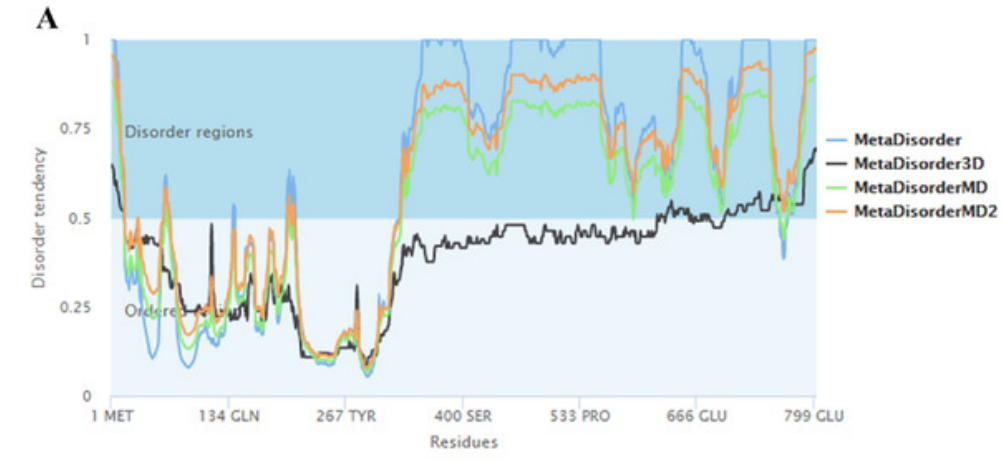

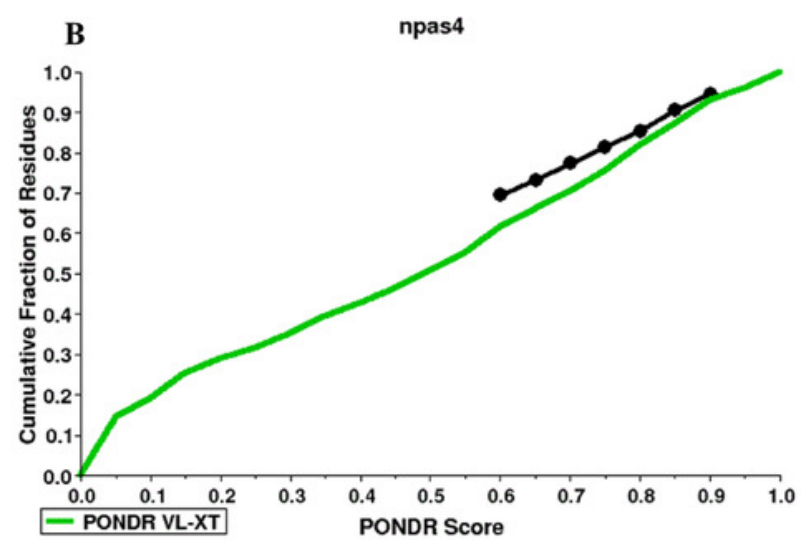




\section{Figure 4}

Amino acid composition of Npas4

(A) Flexibility pattern of amino acids in Npas4 calculated through Composition Profiler. Rigid amino acids are shown in green while flexible amino acids are shown in red. (B) Hydrophobicity of amino acids in Npas4 calculated through Composition Profiler. Hydrophilic amino acids are shown in green color while hydrophobic amino acids are shown in black color.

$\mathbf{A}$

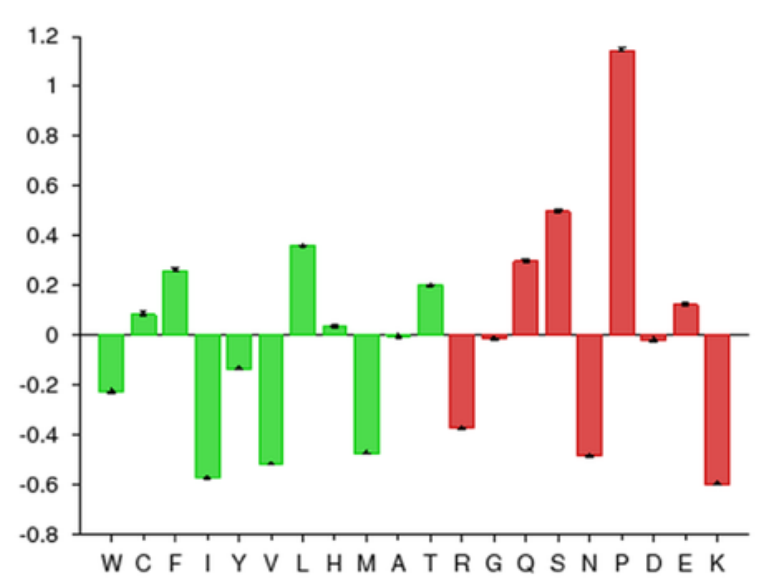

B

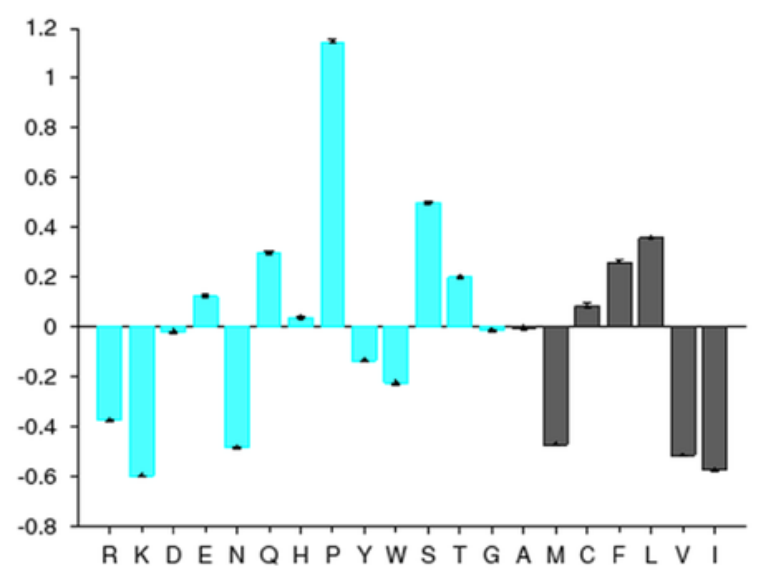


Figure 5

Prediction of physico-chemical properties of Npas4

$\mathrm{X}$-axis shows amino acid sequence from $\mathrm{N}$ - to $\mathrm{C}$-terminal while $\mathrm{Y}$-axis show scores computed by each algorithm. (A) bulkiness; (B) hydrophobicity; (D) polarity; (E) relative mutability.
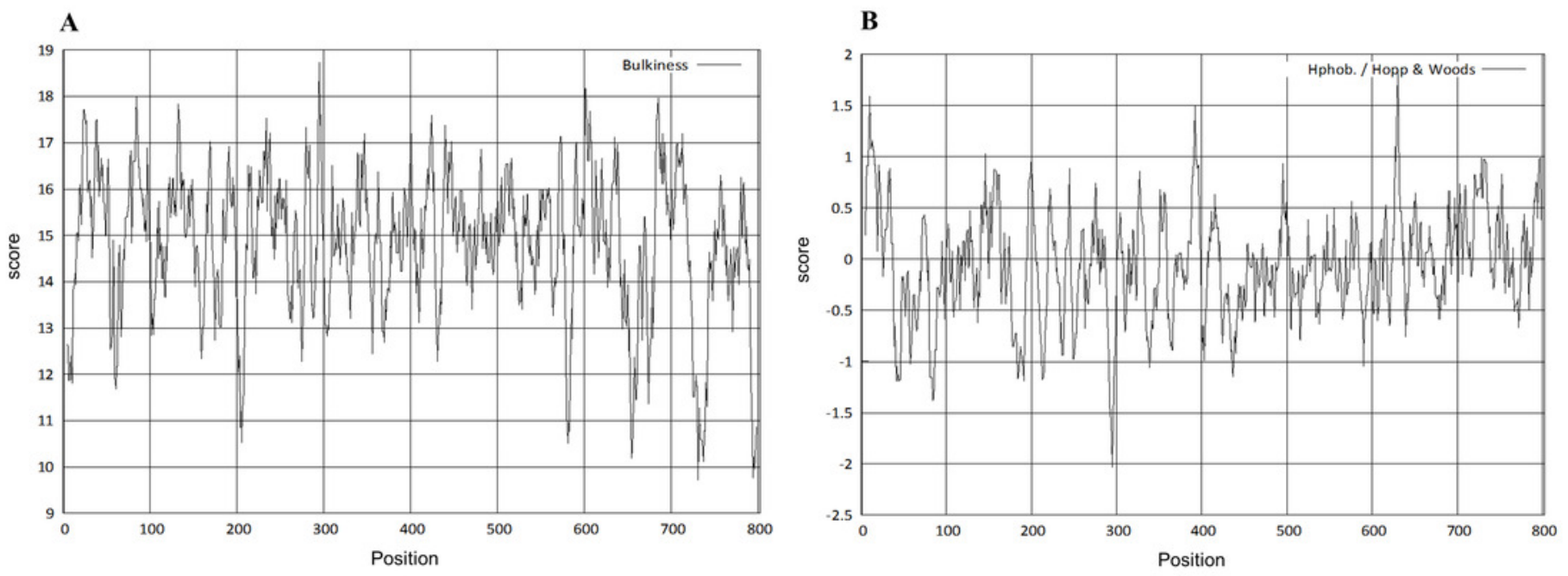

C

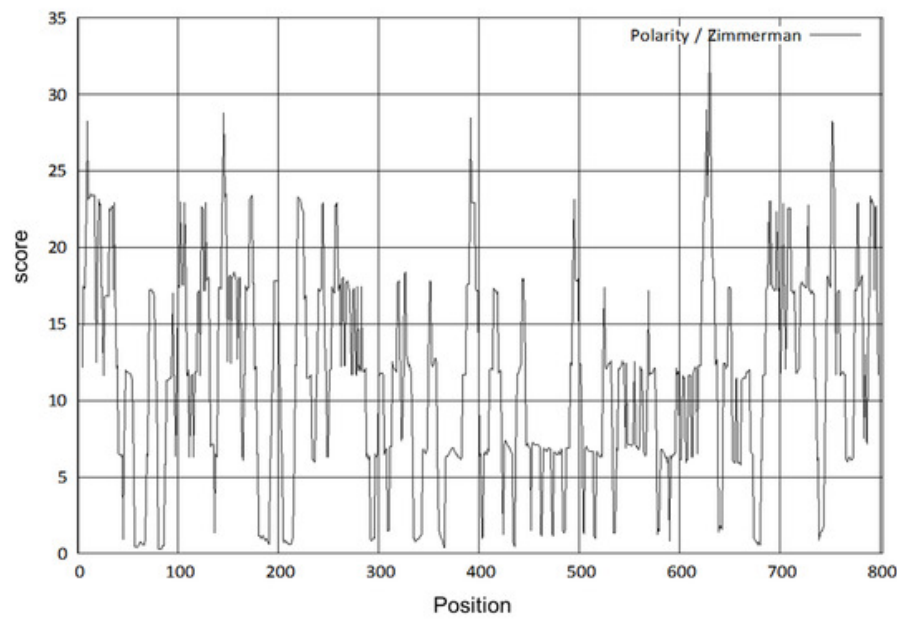

D

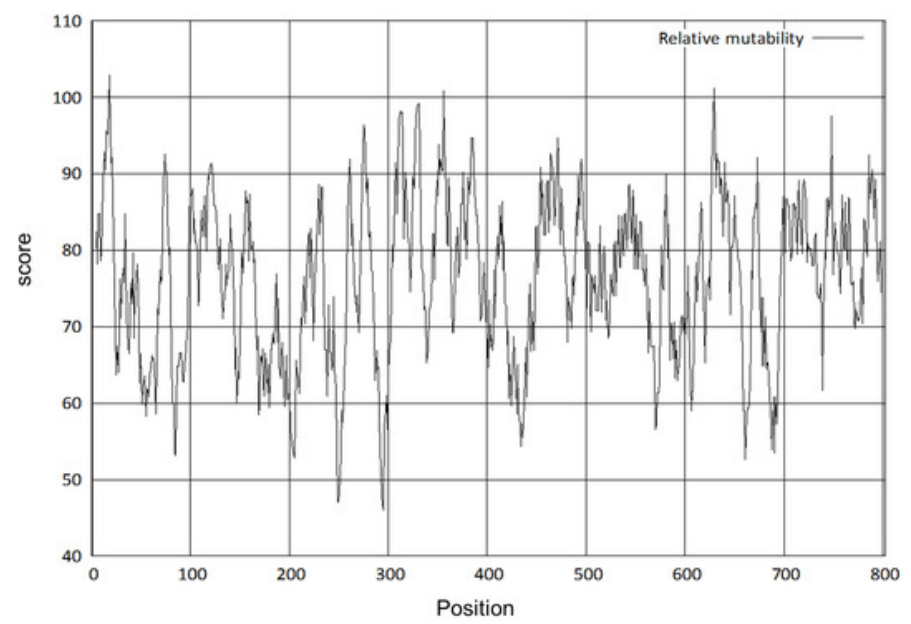




\section{Figure 6}

Prediction of signal peptide of NPAS4 by SignalP server.

$\mathrm{X}$-axis represents amino acid sequence from $\mathrm{N}$ - to $\mathrm{C}$ - terminal while $\mathrm{Y}$-axis represents scores.

Purple line shows the threshold value of 0.5 . The proteins having $C, S$ and $D$ scores above threshold showing the probability of having the signal peptide.

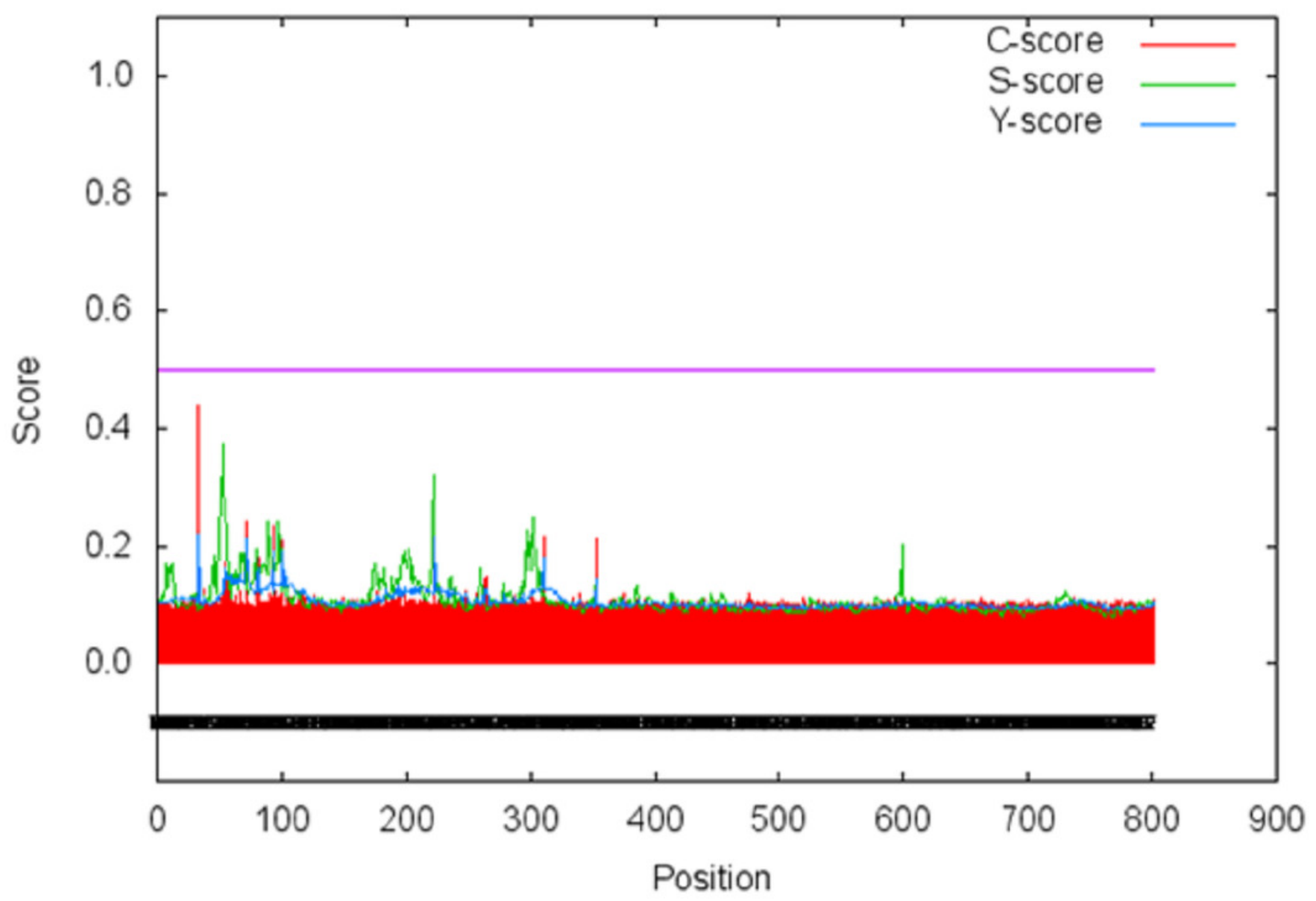




\section{Figure 7}

$\mathrm{N}$-linked glycosylation sites of NPAS4 predicted through NetNGlyc.

$\mathrm{X}$ axis represents amino acid sequence from $\mathrm{N}$ - to $\mathrm{C}$ - terminal while $\mathrm{Y}$ axis represents scores.

0.5 is the threshold, the amino acid positions above threshold level means having potential of glycosylation.

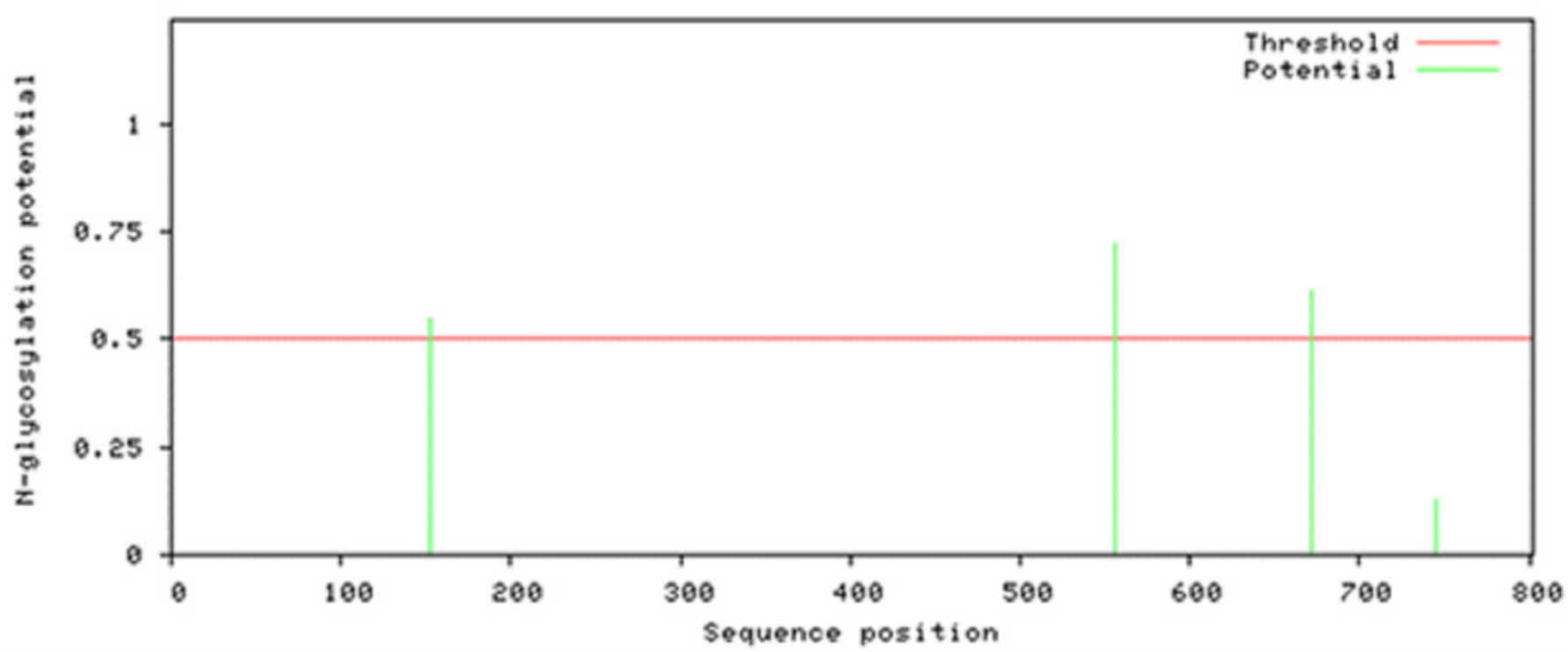




\section{Figure 8}

Potential phosphorylation sites of NPAS4 predicted through NetPhos.

$\mathrm{X}$ axis represents amino acid sequence from $\mathrm{N}$ - to $\mathrm{C}$ - terminal while $\mathrm{Y}$ axis represents scores.

0.5 is the threshold, the amino acid positions above threshold level means having potential of phosphorylation.

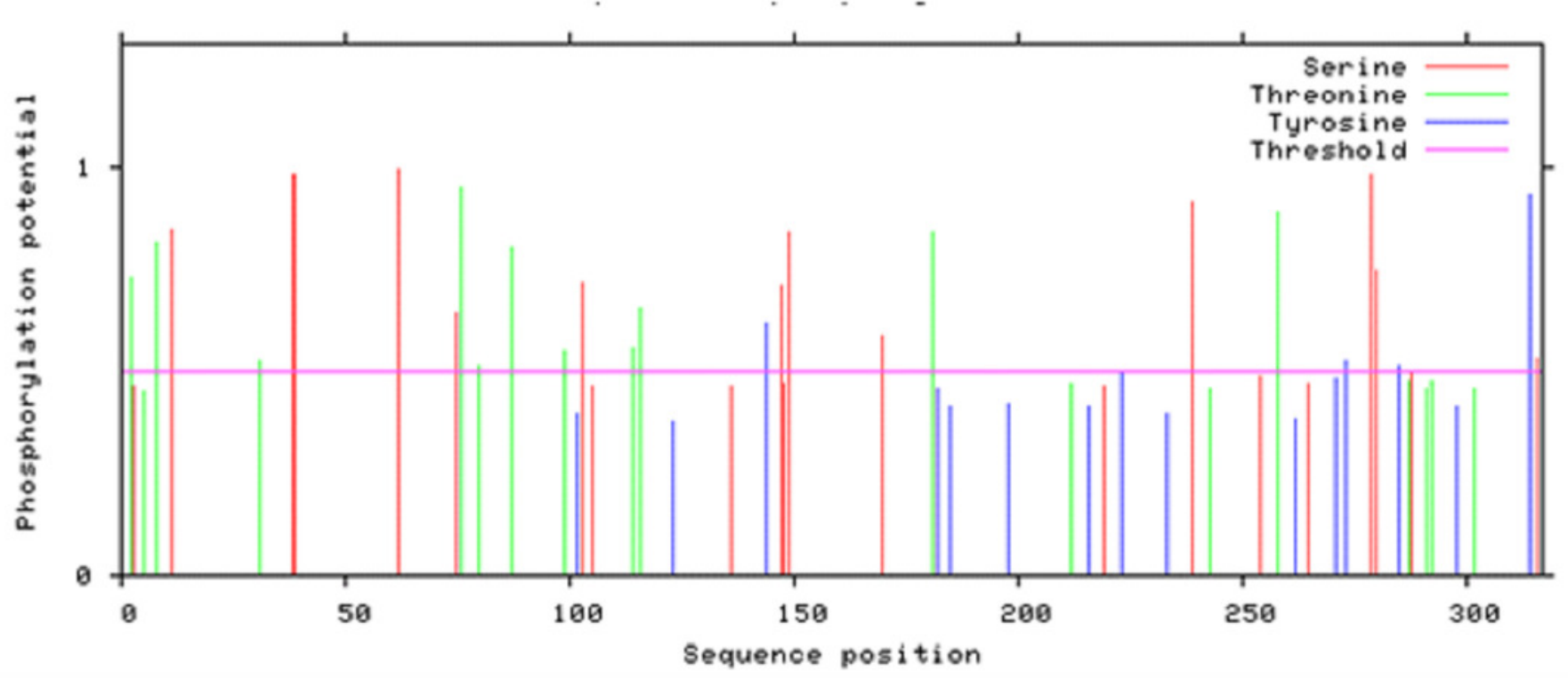


Figure 9

Three dimensional structure of Npas4 predicted through MUSTER and LOMET.

(A) MUSTER-4, (B) MUSTER-5, (C) LOMET-1, (D) LOMET-3, (E) LOMET-4, and (F) LOMET-9. All predicted models show that Npas4 is mixture of order and disorder regions.

A

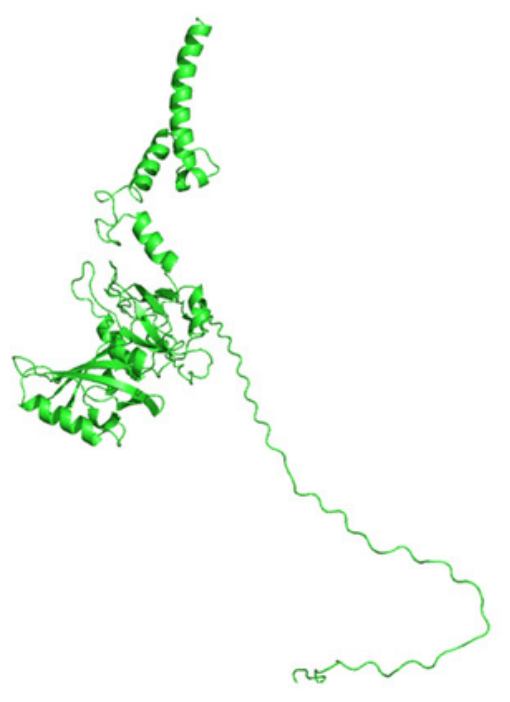

D

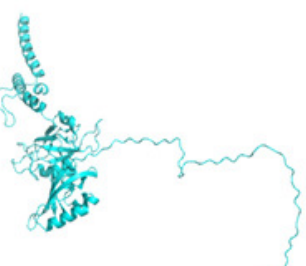

B

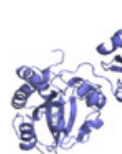

$\mathbf{E}$

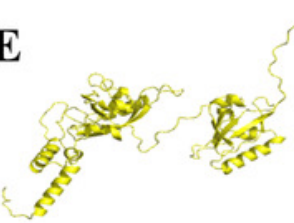

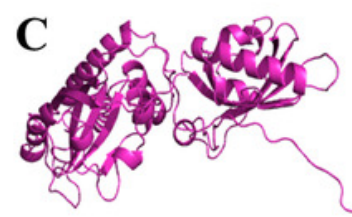

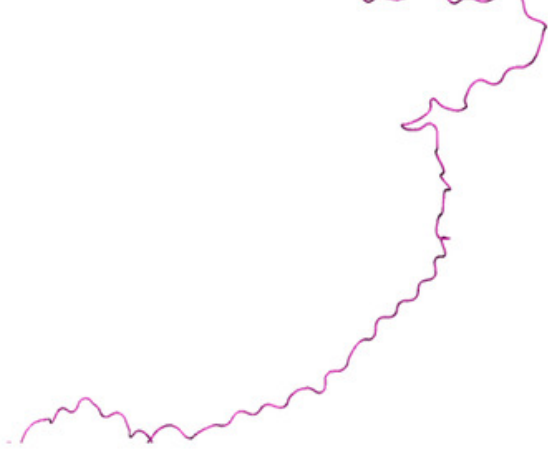

F

$\mathbf{F}$

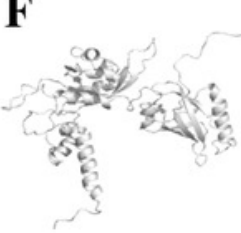




\section{Figure 10}

Posttranslational modifications in bHLH and PAS domains.

bHLH domain is shown in blue (position 10-52 a.a), PAS A domain is shown in yellow (position 72-135 a.a) and PAS B domain is shown in red (position 216-273 a.a). Residues responsible for phosphorylation are highlighted in green.

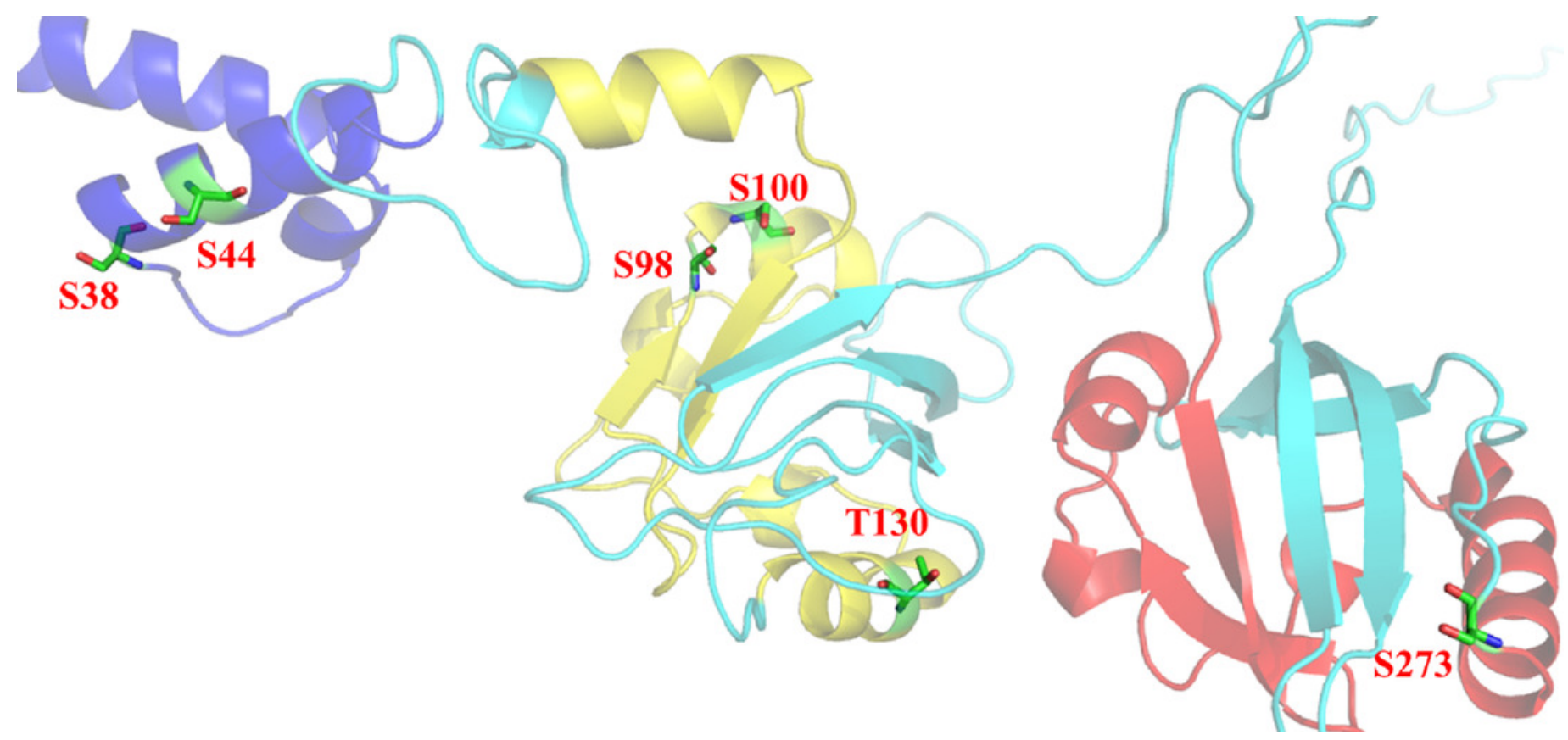




\section{Figure 11}

Interactions of Npas4 with other proteins.

ARNT (Aryl hydrocarbon receptor nuclear translocator); NXF2B (nuclear RNA export factor 2B); NXF2 (nuclear export factor 2); NXF3 (nuclear export factor 3); NDNL2 (necdin-like 2); MAGEF1 (melanoma antigen family F, 1); C3orf58 (chromosome 3 open reading frame 58); NXF1 (nuclear RNA export factor 1); NUP88 (nucleoporin 88KDa); CSAG1 (Chondrosarcoma associated gene 1); MAGEF1 (Melanoma antigen family F, 1); SLC9A6 (Solute carrier family 9, subfamily A (NHE6, cation proton antiporter 6), member 6); MAGED2 (Melanoma antigen family D, 2); SLC9A9 (Solute carrier family 9, subfamily A (NHE9, cation proton antiporter 9), member 9); MAGEL2 (MAGE-like 2); SSX2B (Synovial sarcoma, X breakpoint 2B)SLC9A1 (Solute carrier family 9, subfamily A (NHE1, cation proton antiporter 1), member 1); CREB1 (CAMP responsive element binding protein 1); MAGEA1 (Melanoma antigen family A, 1) \& EPAS1 (Endothelial PAS domain-containing protein 1 ( also known as hypoxia-inducible factor-2alpha (HIF-2alpha)) 


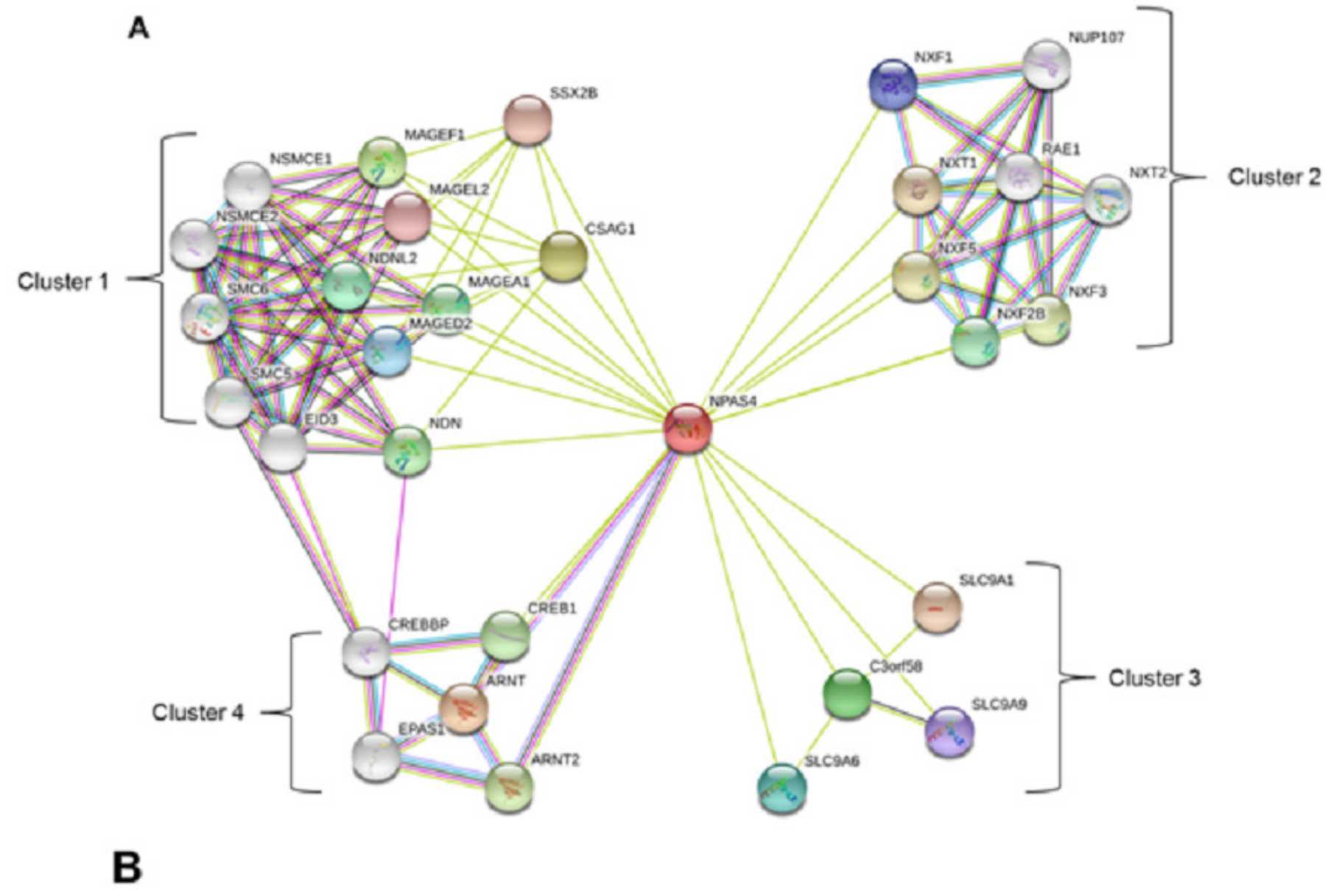

Curated databases

Experimental

Neighborhood

Gene fusion
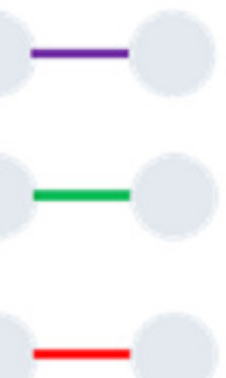

Co-occurrence

Text mining

Co-expression

Protein homology 


\section{Table 1 (on next page)}

Disorder promoting amino acids of Npas4 


\begin{tabular}{|c|c|c|c|c|c|}
\hline \multicolumn{4}{|c|}{ Disorder promoting amino acids } & \multicolumn{2}{c|}{ Order promoting amino acids } \\
\hline Polar/charged amino acids & \multicolumn{2}{c|}{ Small amino acids } & \multicolumn{2}{c|}{ Hydrophobic or bulky } \\
\hline Pro & $10.3 \%$ & Ala & $7.8 \%$ & Leu & $13.0 \%$ \\
\hline Ser & $10.2 \%$ & Gly & $6.6 \%$ & Phe & $4.9 \%$ \\
\hline Glu & $7.2 \%$ & & & Val & $3.2 \%$ \\
\hline Gln & $5.2 \%$ & & & Tyr & $2.6 \%$ \\
\hline Arg & $3.4 \%$ & & & Ile & $2.5 \%$ \\
\hline Lys & $2.4 \%$ & & & Asn & $2.1 \%$ \\
\hline & & & & Cys & $1.6 \%$ \\
\hline & & & & Trp & $0.87 \%$ \\
\hline
\end{tabular}


Table 2 (on next page)

Protein binding regions in NPAS4 


\begin{tabular}{|c|c|c|c|}
\hline \multicolumn{4}{|c|}{ Predicted Disordered Binding Regions } \\
\hline & From & To & Length \\
\hline 1 & 362 & 367 & 6 \\
\hline 2 & 397 & 404 & 8 \\
\hline 3 & 416 & 422 & 7 \\
\hline 4 & 432 & 444 & 13 \\
\hline 5 & 464 & 521 & 58 \\
\hline 6 & 535 & 543 & 9 \\
\hline 7 & 560 & 573 & 14 \\
\hline 8 & 585 & 599 & 15 \\
\hline 9 & 662 & 720 & 59 \\
\hline 10 & 746 & 792 & 47 \\
\hline
\end{tabular}

1 


\section{Table $\mathbf{3}$ (on next page)}

Phosphorylation sites in disorder region of Npas4. 


\begin{tabular}{|c|c|c|}
\hline S.No & Phosphorylation site & Kinases \\
\hline 1 & Ser 611 & P38MAPK, CDK5 \\
\hline 2 & Thr 620 & PKC \\
\hline 3 & Thr 640 & CDC2 \\
\hline 4 & Ser 656 & CKII \\
\hline 5 & Thr 673 & P38MAPK, CKII \\
\hline 6 & Ser 719 & CKII \\
\hline 7 & Ser 728 & CKII \\
\hline 8 & Ser 759 & CKII \\
\hline 9 & Ser 777 & PKC, ATM \\
\hline 10 & Thr 785 & \\
\hline
\end{tabular}

1 
Table 4 (on next page)

Physiological parameters of Npas4. 
1

\begin{tabular}{|c|c|}
\hline Molecular Weight & 87116.58 \\
\hline Theoretical PI & 4.53 \\
\hline Formula & $\mathrm{C}_{3894} \mathrm{H}_{5965} \mathrm{~N}_{1005} \mathrm{O}_{1220} \mathrm{~S}_{23}$ \\
\hline Total no.of Atoms & 12107 \\
\hline Estimated half life & 30 hours \\
\hline
\end{tabular}

2 


\section{Table 5 (on next page)}

Models of Npas4 protein with different methods and their evaluation results. 


\begin{tabular}{|c|c|c|c|c|c|c|}
\hline \multirow[b]{2}{*}{ Model/Tool } & \multirow[b]{2}{*}{ Method } & \multirow[b]{2}{*}{ PROSA } & \multirow[b]{2}{*}{ Qmean } & \multicolumn{3}{|c|}{ Ramachandran plot } \\
\hline & & & & Outlier & $\begin{array}{c}\text { Allowed } \\
\text { region }\end{array}$ & $\begin{array}{c}\text { Favored } \\
\text { region }\end{array}$ \\
\hline I-TASSER-1 & $\begin{array}{l}\text { Multiple- } \\
\text { threading } \\
\text { alignments }\end{array}$ & -3.6 & -11.55 & $9.4 \%$ & $18.6 \%$ & $72.0 \%$ \\
\hline I-TASSER-2 & $\begin{array}{l}\text { Multiple- } \\
\text { threading } \\
\text { alignments }\end{array}$ & -1.28 & -12.31 & $8.4 \%$ & $17.4 \%$ & $74.4 \%$ \\
\hline I-TASSER-3 & $\begin{array}{l}\text { Multiple- } \\
\text { threading } \\
\text { alignments }\end{array}$ & -2.59 & -16.61 & $18.5 \%$ & $28.0 \%$ & $53.5 \%$ \\
\hline I-TASSER-4 & $\begin{array}{l}\text { Multiple- } \\
\text { threading } \\
\text { alignments }\end{array}$ & -4.99 & -16.83 & $19.2 \%$ & $24.6 \%$ & $56.1 \%$ \\
\hline I-TASSER-5 & $\begin{array}{l}\text { Multiple- } \\
\text { threading } \\
\text { alignments }\end{array}$ & -5.07 & -13.55 & $14.2 \%$ & $27.8 \%$ & $58.0 \%$ \\
\hline LEMOT-1 & $\begin{array}{l}\text { Local meta- } \\
\text { threading }\end{array}$ & -2.89 & -3.34 & $1.6 \%$ & $2.2 \%$ & $96.1 \%$ \\
\hline LEMOT-2 & $\begin{array}{l}\text { Local meta- } \\
\text { threading }\end{array}$ & -3.07 & -3.22 & $1.4 \%$ & $2.9 \%$ & $95.8 \%$ \\
\hline LEMOT-3 & $\begin{array}{l}\text { Local meta- } \\
\text { threading }\end{array}$ & -3.66 & -3.08 & $0.8 \%$ & $2.4 \%$ & $96.9 \%$ \\
\hline LEMOT-4 & $\begin{array}{l}\text { Local meta- } \\
\text { threading }\end{array}$ & -3.72 & -3.66 & $1.6 \%$ & $2.4 \%$ & $96.0 \%$ \\
\hline LEMOT-5 & $\begin{array}{l}\text { Local meta- } \\
\text { threading }\end{array}$ & 15.32 & -10.17 & $10.2 \%$ & $14.2 \%$ & $75.6 \%$ \\
\hline LEMOT-6 & $\begin{array}{l}\text { Local meta- } \\
\text { threading }\end{array}$ & -3.11 & -3.96 & $2.0 \%$ & $5.9 \%$ & $92.1 \%$ \\
\hline LEMOT-7 & $\begin{array}{l}\text { Local meta- } \\
\text { threading }\end{array}$ & -2.61 & -2.93 & $1.6 \%$ & $4.5 \%$ & $93.9 \%$ \\
\hline LEMOT-8 & $\begin{array}{l}\text { Local meta- } \\
\text { threading }\end{array}$ & -3.33 & -2.75 & $1.5 \%$ & $2.9 \%$ & $95.6 \%$ \\
\hline LEMOT-9 & $\begin{array}{l}\text { Local meta- } \\
\text { threading }\end{array}$ & -3.63 & -2.61 & $0.6 \%$ & $3.2 \%$ & $96.1 \%$ \\
\hline LEMOT-10 & $\begin{array}{l}\text { Local meta- } \\
\text { threading }\end{array}$ & 3.09 & -20.65 & $7.5 \%$ & $15.9 \%$ & $76.7 \%$ \\
\hline
\end{tabular}




\begin{tabular}{|c|c|c|c|c|c|c|}
\hline RaptorX & Threading & -5.42 & -5 & $2.8 \%$ & $4.7 \%$ & $92.5 \%$ \\
\hline MUSTER-1 & $\begin{array}{l}\text { Multi- } \\
\text { Source } \\
\text { Threading }\end{array}$ & -1.57 & -12.86 & $4.5 \%$ & $8.2 \%$ & $87.2 \%$ \\
\hline MUSTER-2 & $\begin{array}{l}\text { Multi- } \\
\text { Source } \\
\text { Threading }\end{array}$ & -1.51 & -20.75 & $6.2 \%$ & $12.2 \%$ & $81.5 \%$ \\
\hline MUSTER-3 & $\begin{array}{l}\text { Multi- } \\
\text { Source } \\
\text { Threading }\end{array}$ & 1.09 & -2.38 & $7.2 \%$ & $11.2 \%$ & $81.2 \%$ \\
\hline MUSTER-4 & $\begin{array}{l}\text { Multi- } \\
\text { Source } \\
\text { Threading }\end{array}$ & -3.19 & -3.91 & $1.8 \%$ & $1.9 \%$ & $96.4 \%$ \\
\hline MUSTER-5 & $\begin{array}{l}\text { Multi- } \\
\text { Source } \\
\text { Threading }\end{array}$ & -3.35 & -2.88 & $1.1 \%$ & $2.6 \%$ & $96.2 \%$ \\
\hline MUSTER-6 & $\begin{array}{l}\text { Multi- } \\
\text { Source } \\
\text { Threading }\end{array}$ & -2.87 & -20.70 & $4.8 \%$ & $10.0 \%$ & $85.2 \%$ \\
\hline MUSTER-7 & $\begin{array}{l}\text { Multi- } \\
\text { Source } \\
\text { Threading }\end{array}$ & -0.34 & -14.63 & $4.8 \%$ & $9.5 \%$ & $85.8 \%$ \\
\hline MUSTER-8 & $\begin{array}{l}\text { Multi- } \\
\text { Source } \\
\text { Threading }\end{array}$ & -2.44 & -4.14 & $2.1 \%$ & $3.4 \%$ & $94.5 \%$ \\
\hline MUSTER-9 & $\begin{array}{l}\text { Multi- } \\
\text { Source } \\
\text { Threading }\end{array}$ & 0.53 & -9.44 & $7.1 \%$ & $12.0 \%$ & $80.9 \%$ \\
\hline MUSTER-10 & $\begin{array}{l}\text { Multi- } \\
\text { Source } \\
\text { Threading }\end{array}$ & -1.46 & -12.05 & $4.5 \%$ & $12.9 \%$ & $82.6 \%$ \\
\hline Phyre & $\begin{array}{l}\text { Homology } \\
\text { detection } \\
\text { method }\end{array}$ & -4.13 & -8.70 & $7.9 \%$ & $12.8 \%$ & $79.4 \%$ \\
\hline SPARSK-1 & $\begin{array}{c}\text { Fold } \\
\text { Recognition }\end{array}$ & -3.9 & -9.59 & 5.5 & 7.8 & $86.7 \%$ \\
\hline SPARSK-2 & $\begin{array}{c}\text { Fold } \\
\text { Recognition }\end{array}$ & -4.57 & -6.67 & 2.2 & 3.9 & $93.9 \%$ \\
\hline SPARSK-3 & $\begin{array}{c}\text { Fold } \\
\text { Recognition }\end{array}$ & -4.02 & -6.12 & 1.5 & 3.6 & $94.9 \%$ \\
\hline SPARSK-4 & $\begin{array}{c}\text { Fold } \\
\text { Recognition }\end{array}$ & -5.58 & -6.05 & 1.6 & 6.1 & $92.2 \%$ \\
\hline SPARSK-5 & $\begin{array}{c}\text { Fold } \\
\text { Recognition }\end{array}$ & -5.33 & -7.03 & 1.8 & 4.4 & $93.9 \%$ \\
\hline SPARSK-6 & $\begin{array}{c}\text { Fold } \\
\text { Recognition }\end{array}$ & -4.77 & -7.39 & 2.2 & 4.2 & $93.5 \%$ \\
\hline
\end{tabular}




\begin{tabular}{|c|c|c|c|c|c|l|}
\hline SPARSK-7 & $\begin{array}{c}\text { Fold } \\
\text { Recognition }\end{array}$ & -4.05 & -6.53 & 1.4 & 3.6 & $95.0 \%$ \\
\hline SPARSK-8 & $\begin{array}{c}\text { Fold } \\
\text { Recognition }\end{array}$ & 0.7 & -11.0 & 6.6 & 7.4 & $86.0 \%$ \\
\hline SPARSK-9 & $\begin{array}{c}\text { Fold } \\
\text { Recognition }\end{array}$ & -4.3 & -7.51 & 2.4 & 3.6 & $94.0 \%$ \\
\hline SPARSK-10 & $\begin{array}{c}\text { Fold } \\
\text { Recognition }\end{array}$ & -4.71 & -6.99 & 1.8 & 5.0 & $93.2 \%$ \\
\hline
\end{tabular}

2 
Table 6 (on next page)

The validated parameters for refined models of Npas4 Protein. 
1

\begin{tabular}{|c|c|c|c|c|c|c|}
\hline \multirow[b]{2}{*}{ Model/Tool } & \multirow[b]{2}{*}{ Method } & \multirow[b]{2}{*}{ PROSA } & \multirow[b]{2}{*}{ Qmean } & \multicolumn{3}{|c|}{ Ramachandran plot } \\
\hline & & & & Outlier & $\begin{array}{c}\text { Allowed } \\
\text { Region }\end{array}$ & $\begin{array}{c}\text { Favored } \\
\text { Region }\end{array}$ \\
\hline MUSTER-4 & $\begin{array}{c}\text { Multi-Source } \\
\text { Threading }\end{array}$ & -3.19 & -3.91 & $1.8 \%$ & $1.9 \%$ & $96.4 \%$ \\
\hline MUSTER-5 & $\begin{array}{c}\text { Multi-Source } \\
\text { Threading }\end{array}$ & -3.35 & -2.88 & $1.1 \%$ & $2.6 \%$ & $96.2 \%$ \\
\hline LEMOT-1 & $\begin{array}{l}\text { Local meta- } \\
\text { threading }\end{array}$ & -2.89 & -3.34 & $1.6 \%$ & $2.2 \%$ & $96.1 \%$ \\
\hline LEMOT-3 & $\begin{array}{l}\text { Local meta- } \\
\text { threading }\end{array}$ & -3.66 & -3.08 & $0.8 \%$ & $2.4 \%$ & $96.9 \%$ \\
\hline LEMOT-4 & $\begin{array}{l}\text { Local meta- } \\
\text { threading }\end{array}$ & -3.72 & -3.66 & $1.6 \%$ & $2.4 \%$ & $96.0 \%$ \\
\hline LEMOT-9 & $\begin{array}{l}\text { Local meta- } \\
\text { threading }\end{array}$ & -3.63 & -2.61 & $0.6 \%$ & $3.2 \%$ & $96.1 \%$ \\
\hline
\end{tabular}

2 